\title{
The 1957 quantum gravity meeting in Copenhagen: An analysis of Bryce S. DeWitt's report
}

\author{
Alexander Blum ${ }^{1, a}$ and Thiago Hartz ${ }^{2,3}$ \\ 1 Max-Planck-Institut für Wissenschaftsgeschichte, Berlin, Germany \\ ${ }^{2}$ Museu de Astronomia e Ciências Afins, Rio de Janeiro, Brazil \\ 3 Niels Bohr Archive, Copenhagen, Denmark
}

Received 9 March 2017 / Accepted 15 March 2017

Published online 1 June 2017

(C) The Author(s) 2017. This article is published with open access at Springerlink.com

\section{Introduction}

Between June 15 and July 15, 1957, three physicists met at the Institute for Theoretical Physics - the renowned institute directed by Niels Bohr in Copenhagen, Denmark - in order to discuss quantum theories of the gravitational field. They were Bryce S. DeWitt, Stanley Deser, and Charles W. Misner. During the last eleven days of the meeting, they were joined by three other physicists, namely, Christian Møller, Oskar Klein, and Bertel Laurent.

As the meeting had been partially funded by the US Air Force, when DeWitt returned home, to North Carolina, he had to write a research report to the Wright Air Development Center - the US Air Force's research and development center located at the Wright-Patterson Air Force Base in Ohio. Such a report could go into quite some technical detail, as the Wright Air Development Center (or more specifically, the Aeronautical Research Laboratories) had recently hired a bona fide specialist, Joshua N. Goldberg, who was involved in funding and supporting many general-relativityrelated activities from 1956 to 1963 (Goldberg 1992).

DeWitt finished the first version of the report, which has 9 pages, on 31 July 1957; the second and final version, which was considerably enlarged and incorporated comments from the other participants, was completed on 8 October 1957. As far as we know, there is only one remaining copy of the first version of the report and two remaining copies of the second version, one handwritten and one typeset. The first version of the document and the handwritten copy of the second version can be found in possession of Cécile DeWitt-Morette at the University of Texas at Austin. The typed copy of the second version of the document is deposited at the Aage Petersen Collection of Reprints and Manuscripts at the Niels Bohr Library and Archives of the American Institute of Physics, College Park, MD, United States. This last version

a e-mail: ablum@mpiwg-berlin.mpg.de 
is the one that is reproduced in Eur. Phys. J. H, Doi: 10.1140/epjh/e2017-80016-0 and that we introduce and comment on in the next pages.

The relevance of DeWitt's report lies in the amount of background information it gives on the development of quantum gravity at this critical moment: Indeed, one can say that quantum gravity emerges as a research field in its own right at just this time. The Copenhagen workshop is the first scientific meeting dedicated solely to the problem of quantum gravity. And despite the very limited attendance, the report shows the interactions between various approaches and attitudes to the problem and can thereby also serve as an inspiration for today's highly segregated quantum gravity communities. In order to appreciate the full relevance of the report, it is important to have a clear picture of the development of quantum gravity until 1957 . That is the goal of Section 2, where we give a broad panorama of the research on quantum gravity before 1957 and also introduce the actors and the tradition of larger conferences in general relativity in which the Copenhagen workshop is to be situated. In Section 3, we reconstruct the planning of the 1957 meeting. In Section 4 - which is the main part of the article - we present a careful analysis of DeWitt's 1957 report. Finally, in Section 5 we offer our conclusions.

\section{General historical context}

The broad historical context in which the Copenhagen workshop must be placed is the renaissance of general relativity in the 1950s. Recently, a growing number of historians are improving our understanding of this transition, from the marginalization of general relativity between the mid-1920s and the mid-1950s to the establishment of a consolidated area of research in the late 1950s. It is now clear that scientific, institutional, and generational aspects played a role in the establishment of the new community (Blum et al. 2015). In this section, we present those aspects that are most germane to quantum gravity in general and to our story in particular.

\subsection{Research on quantum gravity before 1957}

Before discussing the history of "quantum gravity", we need to briefly discuss our use of this term. It is, flat out, anachronistic. There is so far no historical analysis of the question when, how, and why the term arose, but it most certainly arose years after the Copenhagen meeting and is entirely absent from the report ${ }^{1}$. We use it merely for convenience - the authors' and the readers' - and take it to be synonymous with the "quantization of the gravitational field" of the report's title. This means, in particular, that it does not cover any kind of more general quantum theory that is supposed to reduce to a quantization of general relativity in some lowenergy limit, such as modern-day string theory. Such theories were not yet on the horizon in 1957 and they would not have been deemed necessary: The use of terms such as "quantum gravidynamics" clearly shows that the quantization of gravity was still thought to be essentially analogous to the quantization of electrodynamics, i.e., quantum electrodynamics, as the few scattered early works on quantum gravity had not yet revealed the full extent of the conceptual and formal difficulties involved. In the following we will briefly discuss these few scattered works ${ }^{2}$.

\footnotetext{
1 This is borne out by a simple Google Ngram search, which finds the first usage of the term "quantum gravity" in 1969. Interestingly, in the 1990s, the frequency of "quantum gravity" then already surpasses that of "quantum electrodynamics".

2 The interested reader is referred to a volume currently in press by Dean Rickles and one of the authors which presents early sources in quantum gravity along with a historical contextualization (Blum and Rickles 2017).
} 
When quantum mechanics and quantum field theory had been originally developed in the second half of the 1920s, there was only one quantization procedure, the quantization procedure, namely canonical quantization. When Léon Rosenfeld, then assistant of Wolfgang Pauli, one of the founders of the quantum theory of fields, made the first short-lived forays into the quantization of general relativity in 1930, he naturally applied this quantization procedure both when discussing the gauge invariance properties of the full theory (Rosenfeld 1930a) and when calculating actual toy problems in the linearized (or weak-field) approximation (Rosenfeld 1930b), where the metric is split up into $g_{\mu \nu}=\eta_{\mu \nu}+h_{\mu \nu}$, where $\eta_{\mu \nu}$ is the Minkowski metric and $h_{\mu \nu}$ is taken to be a (small) perturbation ${ }^{3}$.

In the ensuing decades, new quantization procedures developed, not necessarily, however, because of an interest in the foundations of quantum mechanics, but rather with a specific goal in mind, namely to cure the problems of quantum field theory, in the years before the development of renormalization procedures gave a handle on the problematic infinities. Almost invariably, such new procedures would be applied to the quantization of gravity within a few years. A case in point is a quantization procedure originally developed by Dirac (1932); it does not have a real name of its own, because in a sense it is simply equivalent to canonical quantization in the interaction picture (which Dirac introduced for the first time in the 1932 paper). It has, however, the advantage of being manifestly covariant and the disadvantage of only being applicable in the interaction picture, thereby necessitating a clean separation into a free field and perturbing interactions. We might thus call it covariant perturbative quantization. It consists of imposing covariant commutation relations on the free (i.e., interaction picture) field operators at two distinct space-time points, as opposed to the equal-time canonical commutation relations of canonical quantization.

The use of the interaction picture implied a severe restriction in the application of covariant perturbative quantization to gravity: It always had to rely on some sort of perturbation around a flat, Minkowski metric. Covariant perturbative quantization was first applied to linearized general relativity in the 1930s (Bronstein 1936) ${ }^{4}$. But this quantization procedure really only took off when it became the basis for the development of renormalized quantum electrodynamics in the late 1940s and thereby became the standard quantization technique within the high-energy nuclear/particle physics community ${ }^{5}$. In the wake of this great success, a number of physicists conjectured that a full theory of (covariant perturbative) quantum gravity could be constructed by, when expanding the metric in terms of the perturbation $h_{\mu \nu}$, keeping not only the linear term (as Bronstein had done), but also the infinite sum of higher-order, non-linear terms ${ }^{6}$. This was first proposed by Bryce DeWitt (1949) in his unpublished

\footnotetext{
${ }^{3}$ On Rosenfeld's pioneering work, see also (Salisbury and Sundermeyer 2016).

4 A translation of this paper was printed as a "Golden Oldie" in General Relativity and Gravitation, see (Deser and Starobinsky 2012).

5 See, e.g., (Tomonaga 1946) and (Schwinger 1948).

6 This is closely connected to, though logically distinct from, the program of constructing the full theory of general relativity by starting from a free, massless spin 2 field in Minkowski space (first identified with a graviton by Fierz and Pauli (1939)) and then iteratively adding non-linear self-interaction terms through demands of self-consistency. Such a program was first pursued by Robert Kraichnan in his unpublished Bachelor's thesis (MIT, 1947). The thesis is not extant (it is not in possession of the MIT library nor of Robert Kraichnan's widow), and all we have to go by is the abstract of the thesis, which was kindly provided to the authors by Professor John Preskill of Caltech. But in a letter to his thesis advisor Hermann Feshbach (dated 11 July 1947, half a year after submitting his thesis), Kraichnan still could only assert: "I strongly suspect that the physical results of this formalism are identical or essentially similar to those of the general theory of relativity, but I haven't yet been able to get results one way or another". (The letter was in possession of Kraichnan's widow Judy
} 
$\mathrm{PhD}$ thesis and then again by Suraj N. Gupta (1952a,b). This flat-space view was widely accepted in the high-energy community, as one can see from a remark made by Murray Gell-Mann - a leader of that community by the late 1950s - to Gregory Breit in June 1959 that he thought "very well of the Gupta-Feynman view regarding General Relativity with successive correction terms for a Spin 2 particle" 7 . This point of view only began to be called into question several years after the Copenhagen workshop, when Richard Feynman (1963) for the first time actually included the nonlinear terms in an actual calculation, initiating a research program that culminated in the proof that perturbative quantum gravity is non-renormalizable and thus in the realization that the naive covariant perturbative quantization of general relativity does not work.

But already in the 1950s, the perturbative approach was far from uncontroversial and Gell-Mann's opinion was hardly universally accepted. There was the old guard who found the simple, formulaic unification of general relativity and quantum theory presented by Gupta to be a rather bland coda to the great conceptual upheavals of the early twentieth century ${ }^{8}$. But more important for our story is the opposition from the (at least somewhat) younger generation of "relativists" that led the renaissance of general relativity. They felt that the perturbative flat-space formulation was bound to lose essential aspects of the complex non-linear field theory that was general relativity. Indeed, the conviction that general relativity is in some sense special is probably one of the defining new beliefs of the renaissance period. The so-called low-water mark period of general relativity (from the mid-1920s to the mid-1950s) that preceded the renaissance was characterized by a "neo-Newtonian" interpretation (Eisenstaedt 1986 , p. 148-158) of general relativity, which viewed the theory as only providing a small correction to Newtonian physics. In a similar vein, the perturbative approach to (quantum) gravity provided only small corrections to the flat Minkowski space-time of special relativity. This is to be contrasted with the point of view that gained traction in the renaissance of the $1950 \mathrm{~s}$, that especially the non-linearities of general relativity are the essential feature of the theory and lead to properties that are qualitatively different from both Newtonian gravity and special relativity.

One result that strongly promoted this point of view and greatly influenced some of the main proponents of quantum gravity in the renaissance had already been obtained in the late 1930s by Albert Einstein, Leopold Infeld, and Banesh Hoffmann (Einstein et al. 1938). They had shown that the gravitational field equations for empty space are sufficient to derive the equations of motion for a set of gravitationally interacting point-like masses (singularities). Eleven years later, Infeld and Schild (1949) were similarly able to prove the even more elementary result that the vacuum field equations determined the (geodesic) motion of a single point-like test particle in a given background field. These results were in stark contrast to the case of Maxwell

Moore Kraichnan, who kindly provided us with a copy. It is now with the Linda Hall Library for Science, Technology and Engineering, in Kansas City, MO.) His iterative derivation of general relativity was only published almost a decade later (Kraichnan 1955). By this time, the program had been proposed independently by Gupta (1954), who, however, only provided a heuristic sketch of a derivation in order to explain the analogies and disanalogies between his perturbative approach to quantum gravity and quantum electrodynamics. Nowadays, the constructive spin 2 program is mainly associated with Feynman, who based his lectures on gravitation on it (Feynman et al. 1995). Many years later, Deser (1987) argued that, in order to get the Einstein equations with cosmological constant, one could not simply start from a flat space background, but instead had to take an "a priori arbitrary background geometry".

7 Gregory Breit Papers, Yale University, Notes on Yale Theoretical Physics Seminar.

8 See, e.g., Wolfgang Pauli's letter to to Homi J. Bhabha, 26 July 1952, reprinted in (von Meyenn 1996, p. 682), where he describes Gupta's papers as "less interesting", despite the fact that they purported to solve a problem he had a longstanding interest in. 
electrodynamics, where the Lorentz force cannot be derived from the vacuum Maxwell equations. This strongly suggested that the non-linear field theory such as general relativity could provide novel insights into the longstanding problem of integrating the dynamics of continuous fields with those of singular point particles which an essentially linear theory such as electrodynamics could never aspire to.

Following this lead, a new approach to quantum gravity was inaugurated by Peter Bergmann in the late 1940s. Dismissing the modern covariant quantization techniques, which treated the non-linearity of general relativity as mere perturbations, he proposed a return to a canonical quantization of the full non-linear theory. He envisaged a method for transforming the full non-linear Lagrangian into a Hamiltonian, which should then be (canonically) quantized, preserving in the quantum theory the essential features of the non-linearity of general relativity. The problem was to formulate a general method for obtaining a Hamiltonian from a Lagrangian with gauge symmetries, i.e., from the Lagrangian of a constrained system. In quantum electrodynamics and in the linearized gravitational theory, methods for dealing with this difficulty had been developed in the 1930s. It was, however, far from clear how to extend these methods to the non-linear case. The solution to this problem was developed independently by Peter Bergmann and his students (Bergmann et al. 1950), and by Pirani and Schild (1950), building on work by Paul Dirac (1950) and Paul Weiss (1938). That was the beginning of the canonical quantum gravity program. As opposed to the perturbative covariant approach, which in 1957 was viewed as either completed or intrinsically insufficient, canonical quantization of gravity was an active research program in 1957 and was extensively discussed at the Copenhagen workshop (Sect. 4.4).

While Bergmann proposed a return to the roots of quantum mechanics and the original canonical quantization procedure, new quantization techniques were proliferating. Misner, in an introductory passage - hailed by Carlo Rovelli (2004, Appendix B) as a moment of clarity in the history of quantum gravity - to his 1957 thesis on quantum gravity asserted that

Four approaches have been suggested to discover the content of the quantum theory of general relativity... (Misner 1957, p. 497)

Besides the already mentioned canonical and perturbative covariant approaches, this list also included quantization using Schwinger's action principle and Feynman (or path integral, in modern parlance) quantization. And even this list was not exhaustive, omitting lesser known methods such as Peierls brackets.

The most prominent of the new quantization techniques was, however, certainly Feynman quantization, which was also the central focus of Misner's thesis. It had been developed by Feynman in the early 1940s, in an attempt to quantize classical theories which were not defined by a Hamiltonian or Lagrangian density, but only by an action. Such a classical theory had been devised by Wheeler and Feynman (1945) in order to eliminate both the infinite number of degrees associated with the electromagnetic field and the problematic self-interaction of charged matter, both of which were viewed as the classical origin of the divergence difficulties of quantum electrodynamics. Just like perturbative covariant quantization, Feynman quantization had thus been developed in the context of attempting to cure the woes of quantum field theory more generally. While Feynman never managed to quantize Wheeler-Feynman electrodynamics using his path-integral approach, his investigations did lead to the development of this new quantization method, which not only gave an interesting novel view of non-relativistic quantum mechanics, but also was of immense heuristic value in establishing the Feynman rules for regular quantum electrodynamics ${ }^{9}$. The idea to use Feynman quantization to quantize gravity was only a couple of years

${ }^{9}$ On the genesis of Feynman quantization, see, e.g., (Wüthrich 2010) 
old in 1957 and had not progressed very far, but among the Copenhagen workshop participants it was probably the most popular approach overall (see Sect. 4.3).

\subsection{Participants of the copenhagen meeting}

The 1957 meeting took place in Copenhagen and thus it is no wonder that three of the participants (those three that only joined for the latter part) came from what might be called the Scandinavian tradition in general relativity. In Northern Europe, there had been persisting interest in general relativity from a few physicists since the late 1920 s.

Christian Møller had become well-known due to his work on scattering theory - both electron-electron scattering and meson scattering - during the 1930s and 40s. He lectured at the Institute for Theoretical Physics in Copenhagen from the early 1930s until his death, in 1980 (Brevik 2011; Rozental 1985, p. 8). In the mid-1940s, he engaged himself in Heisenberg's S-matrix program, but put it aside to ${ }^{10}$ write a highly regarded textbook (Møller 1952) based on his general relativity lectures during the 1930s and 40s, which became a classic text in general relativity (Wheeler and Ford 2000, p. 305).

One of the most important aspects in his teaching of general relativity was his attitude towards the four-dimensional formulation. He acknowledged the historical relevance of having recognized the similarities between space and time, but that was, according to him, not the best way of approaching the teaching of general relativity. Instead, he asserted one should "stress again the fundamental physical difference between space and time, which was somewhat concealed by the purely formal fourdimensional representation" (Møller 1952, p. v-vi). Such a program was envisaged for pedagogical reasons:

The three dimensional point of view (...) leads to a reintroduction of dynamical concepts into the gravitational theory, which, I believe, makes it easier for the student fully to grasp the physical content of the general theory of relativity

(Møller 1952, p. vi).

These pedagogical perspectives strongly influenced his research agenda, which began focusing on general relativity in the 1950s. He always emphasized the importance of studying how time was to be measured in general relativity and, therefore, dedicated most of his attention to analyzing the behavior of clocks in a gravitational field - as we will discuss in Section 4.1.

Oskar Klein had also been a member of the Copenhagen Institute for Theoretical Physics, from 1918 to 1923 and again from 1925 to 1930. In the latter period, he was Niels Bohr's assistant, as well as a leading figure in theoretical physics himself, obtaining important results such as the Klein-Gordon equation or the Klein-Nishina formula. In 1931 he became professor at Stockholm College (later Stockholm University), where he lectured on general relativity (Feyerabend 1995, p. 77; Fisher-Hjalmars and Laurent 1991). Stanley Deser (1977) observes that Klein was "one of the first who seriously considered general relativity in connection with quantum theory, an interest that remained with him from the time of the formulation of the Klein-Kaluza theory in 1926". After a long period of abstinence, he returned to the problem of quantum gravity in the mid-1950s, outlining the following research program:

As a kind of programme we shall thus put forward the following claim: The operators to be used in quantum field theory should have a simple connection to a transformation group (so far insufficiently known) which contains

\footnotetext{
${ }^{10}$ Letter from Møller to Wolfgang Pauli, 13 November 1946, reprinted in (von Meyenn 1993).
} 
the general coordinate transformations in spacetime as a subgroup. The quantum conditions ought to characterize the group in question. In trying to develop a theory according to such a programme it should be kept in mind that the direct quantization according to the ordinary scheme of quantum mechanics of the Einstein equation meets with difficulties of the same type, but very much enhanced, as those met with in the quantization of the Maxwell equations. Also from this point of view it would seem preferable to start with the quantum conditions expressing group properties instead of starting with a Lagrangian density. This would probably make the theory still more symbolic and remote from direct observation than ordinary quantum field theory (Klein 1955, pp. 98-99).

This research program was further developed by Bertel Laurent. Laurent was Klein's last PhD student and was still working on his thesis on quantum gravity in 1957 (Deser 1995). The thesis, entitled "Studies in the Synthesis of Quantum Theory and General Relativity" was submitted on 5 December 1959.

Klein had also further explored the idea that the gravitational field might work as a regulator for quantum field theory:

It is perhaps not unreasonable that the rigorous consideration of gravitational and perhaps other similar non-linear effects would do away with the remaining divergencies of electron theory. (Klein 1956, p. 61)

This went beyond the brute force regularization using a minimal length scale that had been explored already in the 1930s (Heisenberg 1938), and Klein's statement would have a deep influence on the 1950s generation - as we will discuss in Section 4.5.

The other three participants of the Copenhagen workshop came from the United States. There, general relativity had been practiced by a few physicists in the 1930s and $40 \mathrm{~s}$, to a large part due to European refugees. Until the mid-1950s, these researchers had virtually no interaction. This situation began to change, as several American physicists set up new centers of relativity research in the second half of the 1950s and, above all, due to the 1957 Chapel Hill conference (see Sect. 2.3).

Bryce DeWitt had been a PhD student of Julian Schwinger at Harvard. Schwinger himself was not interested in general relativity until the late 1950s; around 1950, lectures on general relativity at Harvard University were given by Philipp Frank, whose chief interests were the philosophical aspects of the theory (DeWitt 2011, p. 51). Nevertheless, Schwinger did supervise DeWitt's $1949 \mathrm{PhD}$ thesis on self-energy problems related to the quantization of the gravitational field. DeWitt recalled:

I had a strong feeling that Einstein's theory was in a sort of limbo, detached from the rest of physics, and that it was a shame that such a beautiful theory should be so ignored. I proposed to drag it forcibly into the then modern world by redoing Schwinger's QED calculations with the gravitational field added. I was very naive in those days (DeWitt 1996a).

DeWitt's thesis (which relied on the covariant perturbative quantization) exemplifies the initial expectation that a quantum theory of gravity could be constructed by applying methods from QED to the gravitational field. Of course several physicists had already anticipated that such an expectation was unfounded - for instance, Matvei Bronstein - but such warnings could at the time only be based on qualitative, heuristic reasoning ${ }^{11}$. It was only when the works of Bergmann, Dirac, Pirani, and Schild on constrained dynamics appeared, right after DeWitt completed his thesis, that it was widely acknowledged (outside the immediate high-energy/quantum field

11 See (Blum and Rickles 2017). 
theory community) that these results made the Lorentz-covariant perturbative approach to quantum gravity obsolete. In a paper based on his thesis, which DeWitt submitted to the Physical Review ${ }^{12}$, he remarked:

[A] perturbation philosophy is adopted. The linear (zero order) part of the gravitational Lagrangian function is subtracted from the full Lagrangian. The residue, which contains all the self-interaction effects [...] is treated as a perturbation but is never closely examined. This procedure results, of course, in a rather makeshift formulation of the theory [...]

The use of a makeshift formulation has [...] been prompted by a more pressing consideration. At the time of initial writing of these papers no one had yet explicitly constructed a gravitational Hamiltonian for the general theory [of relativity]. [Footnote:] F. A. E. Pirani and A. E. Schild, and independently, P. G. Bergmann and his co-workers [...] have just recently succeeded in constructing the required Hamiltonian function. [...] It may eventually be of interest to reformulate some of the present calculations according to the rigorous PiraniSchild-Dirac scheme.

That such a reformulation would not just "eventually be of interest", but was in fact the only thing of immediate interest, was the view taken by the paper's referee, Howard P. Robertson: "In view of the Pirani-Schild paper, which [DeWitt] has seen - and remarks on p. 3, footnote, that this should eventually be carried out in terms of their more rigorous theory - it would seem to me better to suggest he carry out his work in terms of their theory" ${ }^{13}$.

Bit by bit, the perturbative approach began to be rejected by the general relativity community (in spite of Gupta's concise 1952 formulation of the perturbative theory). DeWitt then was faced with a dilemma. As a student of Schwinger, he had learned to give first priority to explicit covariance; but the Bergmann-Pirani-Schild canonical methods were moving away from explicit covariance and adopting an explicit separation of time and space. At the same time however, the successful covariant methods of QED - such as the Gupta-Bleuler method, the Schwinger action principle, the Peierls bracket, etc - were not directly applicable to the (non-linear) gravitational case. DeWitt dealt with this dilemma by adopting a many-pronged approach to quantum gravity, investigating several quantization methods and their interrelations. This included the canonical approach, but only in its original fully four-dimensionally covariant (so called parameter) form. We will return to this specific point in detail in Section 4.4. DeWitt's inclusive interest in the different quantization programs and his ambition to overcome their respective shortcomings by relating them to one another is a leitmotif of his entire 1957 Copenhagen report.

Between 1950 and 1953, DeWitt had postdoctoral stays (working on quantum gravity) at the Institute for Advanced Study (Princeton), the ETH Zurich, and at the Tata Institute (Bombay) (DeWitt 2011, p. 140). After working on hydrogen bomb development at Livermore Laboratory for a three-year period ${ }^{14}$, he created in 1956 , together with his wife Cécile DeWitt-Morette, the Institute of Field Physics at the University of North Carolina at Chapel Hill, North Carolina (DeWitt and Rickles 2011).

\footnotetext{
12 Preprint of the article "On the application of quantum perturbation theory to gravitational interactions, part I", 1950. Bryce S. DeWitt Personal Files, in Cécile DeWitt-Morette's office, University of Texas at Austin, Austin, TX. Hereafter this collection will be referred to as DF.

${ }^{13}$ Referee report, 17 May 1950. Howard P. Robertson Papers, 1922-1980, box 7, folder 14. California Institute of Technology Archives.

14 As outlined in "Cold War Curvature", a talk given by David Kaiser at "The 'Renaissance' of General Relativity in History: Assessing Einstein's Legacy in Post-World War II Physics", the General Relativity Centenary Conference held in Berlin from 2-5 December 2015.
} 
Cécile DeWitt-Morette did some work on general relativity in the 1950s, but her main areas of expertise at that time were meson theory and Feynman's path integrals. While there were no other physicists actively working on general relativity when the DeWitts came to North Carolina, it was being taught by Eugene Merzbacher, who had also been a PhD student of Schwinger's. Around 1957, DeWitt started training his first graduate students, Robert Brehme (PhD '59), John J. Ging (PhD '60), and Hsin Yang Yeh (PhD '60).

Stanley Deser had also obtained his $\mathrm{PhD}$ with Schwinger. His thesis, completed in 1953, was, however, on meson scattering, i.e., on a topic entirely unrelated to general relativity. Deser then went to the Institute for Advanced Study in Princeton, where he attended a lecture by Albert Einstein and met Elsbeth Klein, the daughter of Oskar Klein, whom he would marry in $1956^{15}$. In 1955, Deser went to the Institute for Theoretical Physics in Copenhagen. Until his arrival in Copenhagen, Deser had not worked on general relativity. According to his recollections, it was after his meeting with Oskar Klein in Bern in 1955 that he started thinking more seriously about the subject:

Our meeting and the [Bern] conference also affected me, and I began to think more seriously about the possibility that general relativity could be a universal regulator of the divergence problems of quantum field theory, and in particular of QED. This idea had also occurred to Landau, Pauli and Oskar himself (Deser 1995, p. 3).

We will return in detail to this research project of Deser's in Section 4.5.

The third American participant of the Copenhagen workshop, Charles Misner, had only recently obtained his $\mathrm{PhD}$ at Princeton under the supervision of John Archibald Wheeler. In 1957, Princeton was arguably the most prestigious center for general relativity in the United States, hosting not only Wheeler but also Eugene P. Wigner, who got involved in general relativity in 1955, when he began to work with Helmut Salecker on the quantum limitations of clocks in general relativity (Salecker and Wigner 1958) - a work deeply related to Møller's, as we will discuss in Section 4.1.

Wheeler had given his first series of lectures on general relativity in Princeton in 1952 (Wheeler and Ford 2000, p. 228) and soon took gravitation to be the cornerstone of his new research program that "everything is field". A paradigmatic example of that program was the geon, an object envisaged by Wheeler in which an electromagnetic wave is held together by its own gravitational field (Wheeler 1955; Hartz and Freire 2015). While a quantum theory of geons was (and is) still far off, Wheeler did invoke heuristic estimates of quantum effects, in order to make it plausible that quantum geons would eventually have the right dimensions for identifying them with elementary particles (as opposed to classical geons, which were much too large).

Wheeler, in his attempts to turn general relativity from an abstract philosophicomathematical field into an integral part of physics, also supported experimental efforts in general relativity, starting in 1955 with Robert H. Dicke and Joseph Weber (see the article by Jim Peebles, Eur. Phys. J. H, Doi: 10.1140/epjh/e2016-70034-0). On the theoretical side, Wheeler's star PhD student of the 1950s was Misner. His 1957 thesis was specifically on the application of Feynman's path integral methods to the quantization of the gravitational field, but also discussed in detail the relation of this approach to other attempts at quantizing general relativity.

The three US physicists present at the 1957 Copenhagen meeting did not represent US-American quantum gravity research in its entirety. We have already mentioned the groups of Peter Bergmann and Alfred Schild. The German-born Bergmann had,

\footnotetext{
${ }^{15}$ See (Halpern 2004, p. 213-214) and a letter from Pauli to Gunnar Källén, 7 October 1956, reprinted in (von Meyenn 2001, p.687-688).
} 
at age 21, become Albert Einstein's assistant in Princeton, a position that he occupied for five years. He later wrote an acclaimed textbook on both special and general relativity (Bergmann 1942). In 1947, he went to Syracuse University, where he created his research group on general relativity and quantum gravity. As two of Bergmann's collaborators observed:

When Peter began his career at Syracuse in 1947, no US physics department had a center for research in general relativity. Indeed, very few physicists considered the area worthy of their time. Within the Syracuse physics department, Peter created one of the first groups specifically concerned with studying the general theory of relativity with the intent of reconciling that field with quantum theory. [...] Up to the mid-1950s, Peter and his students were the major contributors to the literature in general relativity (Goldberg and Schucking 2003).

Schild (also German-born) had obtained his PhD at the University of Toronto in 1946 under Leopold Infeld. He then went to the Carnegie Institute of Technology, in Pittsburgh, where he did his work on canonical quantization together with his PhD student Felix Pirani, who had followed Schild from Toronto. Schild moved to the University of Texas at Austin in 1957, where he created the Center for Relativity five years later (Schucking 1989).

Finally, at Purdue University, the Dutch physicist Frederik J. Belinfante set up a group working on quantum gravity in the 1950s. Belinfante pursued several approaches in parallel, considering both the quantization of alternatives to general relativity (the linear, Lorentz covariant Belinfante-Swihart theory) and the canonical quantization of general relativity itself, in both cases with a strong emphasis on the interaction of gravitation with other fields, especially spinorial matter.

Research explicitly on quantum gravity was, however, not the sole contemporary influence on the Copenhagen workshop. Early on, there was a very strong European mathematical tradition in general relativity (focusing on its relation with differential geometry), associated with the names of Tullio Levi-Civita, Cornelius Lanczos, Theóphile de Donder, Théophile Lepage, Élie Cartan, and Georges Darmois, among others. Some physicists even perceived general relativity as a branch of mathematics (Mercier 1992), and in some countries, in particular in France, this did not change in the 1950s. The doyen of French mathematical relativity in the 1950s was André Lichnerowicz ${ }^{16}$; his interest in quantum gravity began only in $1958^{17}$, but the work of him and his school on the initial value problem (Lichnerowicz 1955) did have some impact on the discussions in Copenhagen, as we will see in Section 4.2.

After this brief overview of the participants of the Copenhagen workshop and the contemporary quantum gravity scene they were moving in, we arrive at the conclusion of this section. While there was a drastic increase in the research on general relativity in the 1950s, there were only a few groups working on quantum theories of the gravitational field. Even though the 1957 meeting in Copenhagen gathered only six physicists, it still represented a significant proportion of researchers interested in quantum gravity, and all the trends in quantum gravity research touched on in the last two sections were actually discussed at the Copenhagen meeting.

\subsection{The first conferences on general relativity}

The establishment of new research groups was not the only institutional advance of the 1950s, which also saw the organization of the first conferences devoted solely to general

\footnotetext{
16 For more on this towering figure, see, e.g., (Kosmann-Schwarzbach 2009).

17 See (Lichnerowicz 1964).
} 
relativity and the creation of the International Committee on General Relativity and Gravitation, which aimed at improving the communication between the researches in the field (see Blum et al. 2015, p. 617).

In 1954, on the eve of the fiftieth anniversary of the creation of special relativity, André Mercier solicited the help of Wolfgang Pauli in co-organizing a conference on the occasion (Mercier 1992). The conference, which had ninety participating scientists and took place in Bern, Switzerland, in July 1955, also turned out to be memorial for the creator of the relativity theories: Albert Einstein had passed away in April, only three months before the conference (Mercier and Kervaire 1956).

The Bern conference has been hailed on various occasions as the most important moment in the renaissance of general relativity. Lichnerowicz (1992, p. 105) writes that "the International Congress at Bern [...] marked the true renaissance of interest in general relativity on the part of physicists, astronomers, and mathematicians". Mercier (1992, p. 119) claimed that there the quantum gravity problem "was asserted with steadfastness that did not leave much to be desired". Eisenstaedt (1986) chose the year 1955 as the end of the "low-water-mark" period.

For all its importance, the Bern conference was not the only central event of the renaissance and its importance is greater for Europe than it is for the United States. As Dean Rickles has pointed out, "the Bern conference consisted mostly of an older generation who had persistently thought about general relativity and quantum gravity for decades". (DeWitt and Rickles 2011, p. 19). In the US, the training of a new (arguably the first) generation of relativists had begun before the Bern conference and the event at which this generation established itself as a force to be reckoned with took place one and a half years after the Bern conference, on 18-23 January 1957, at Chapel Hill. It was organized by Bryce DeWitt and his wife Cécile DeWitt-Morette and was also the official inaugural event of the Institute of Field Physics, that had begun its activities in January 1956. The project was funded by Agnew H. Bahnson, a North Carolina industrialist and philanthropist.

The Bern and the Chapel Hill conferences were unrelated as far as their organizational structure was concerned. However, in order to create some sort of tradition - or even to increase the importance of their event -, Bryce and Cécile DeWitt began to refer to the Chapel Hill conference as the "Second International Conference on Gravity" ${ }^{18}$. In Chapel Hill, Lichnerowicz offered to organize the follow-up conference in Royaumont, France, in 1959 (see Lichnerowicz 1992, p. 91, and Lichnerowicz and Tonnelat 1962). In 1962, the next conference in the series was held in Warsaw, Poland, organized by Leopold Infeld (Ashtekar 2014; Infeld 1964). Nowadays, these conferences are viewed as having inaugurated the still ongoing tradition of GR conferences, counting Bern as GR0, Chapel Hill as GR1, Royaumont as GR2, and Warsaw as GR3.

Those conferences - the amount of new questions that were formulated, the intense discussions among its participants, the huge progress in general relativity from one conference to another - are the greatest historical evidence showing the renaissance of general relativity. Because of the Chapel Hill conference, the year 1957 is well recognized as a turning point in quantum gravity research. According to Rickles:

The Chapel Hill conference on the Role of Gravitation in Physics [...] did for general relativity what Shelter Island did for quantum electrodynamics. The Chapel Hill conference was a genuine break from the Bern conference, both in terms of its organization, its content, but more so its spirit (DeWitt and Rickles 2011, p. 19-20).

The 1957 Copenhagen meeting happened just five months after the Chapel Hill conference and many of the participants in the Copenhagen conference had also been at

\footnotetext{
18 Bahnson Memoranda \#5 (16 October 1956) and \#6 (1 November 1956). DF, box Institute of Field Physics.
} 
Table 1. Who attended each conference?

\begin{tabular}{ccccc}
\hline Person/Conference & \multirow{2}{*}{ Bern '55 } & $\begin{array}{c}\text { Chapel } \\
\text { Hill '57 }\end{array}$ & $\begin{array}{c}\text { Royaumont } \\
\text { '59 }\end{array}$ & $\begin{array}{c}\text { Warsaw } \\
\text { '62 }\end{array}$ \\
\hline Stanley Deser (1931-) & Yes & Yes & Yes $^{19}$ & Yes \\
\hline Bryce S. DeWitt (1923-2004) & No & Yes & Yes & Yes \\
\hline Oskar Klein (1894-1977) & Yes & Yes & No & No \\
\hline Bertel E. Laurent (1928-1993) & No & Yes & Yes & Yes \\
\hline Charles W. Misner (1932-) & No & Yes & Yes & Yes \\
\hline Christian Møller $(1904-1980)$ & Yes & No & Yes & Yes \\
\hline
\end{tabular}

Chapel Hill. In Table 1, we present a list of the six participants of the 1957 Copenhagen meeting with the information on which of the first four major GR conferences they attended. Also thematically, several of the issues discussed in Chapel Hill reappeared in the Copenhagen discussions, as we will highlight systematically in Section 4. However, as opposed to the conferences listed above, the meeting in Copenhagen had a very specific focus: It was the very first gathering of researchers to discuss solely the quantization of gravitation. In the next section, we discuss how this specialized meeting came to be.

\section{The history of the 1957 meeting in copenhagen}

\subsection{Christian Møller and the Research Group in Copenhagen}

Since the establishment of the Institute of Field Physics, Bryce and Cécile DeWitt put a lot of effort into fund raising. Together with Agnew Bahnson, they approached a great number of industrialists and created a fair amount of publicity for their work, mainly through newspaper articles. A fundamental step in drawing general attention to the Institute was the 1957 Chapel Hill conference. It was therefore of central importance to attract well-known physicists of international renown to that event.

DeWitt made a preliminary list of eighteen "physicists whom we definitely want to come [to the Chapel Hill conference] and who would be expected to play an active role in the discussions" 20 . There were five physicists from Europe on the list: Niels Bohr, Christian Møller, André Lichnerowicz, Oscar Klein, and Marie-Antoinette Tonnelat. Apparently concerned with funding restrictions, DeWitt decided to choose just three of them. As far as we know, Bohr never got invited. He had already declined the invitation to attend the 1955 Bern conference, feeling that "he had not thought enough about the possible future of a generally relativistic cosmology that would have given him premonitions of a development to come" (Mercier 1992, p. 112).

Møller had been a key person in the development of the Institute for Theoretical Physics in Copenhagen. Denmark had been one of the candidates for hosting the CERN laboratory in a competition that was ultimately won by Switzerland in October 1952 (Krige 1987a). Møller took part in these discussions as one of the representatives of the Danish government (Pestre 1987, p. 161). As part of Denmark's involvement in the creation of CERN, the CERN Theoretical Study Group was established in Copenhagen in 1952. Møller became the Director of the group in September 1954, taking over a position that had previously been Niels Bohr's (Krige 1987b, p. 217). He kept his position as the director of the research group until it was replaced, in

\footnotetext{
19 Wrongly referred to in the conference proceeding as F. Deser.

${ }^{20}$ Letter from Bryce S. DeWitt to Agnew H. Bahnson Jr., 7 March 1956. DF, box Institute of Field Physics, folder Bahnson Correspondence.
} 
early September 1957, by a new Theoretical Study Group in Geneva, marking the end of CERN activities in Copenhagen (Krige 1987a, p. 246). Nevertheless, the staff of the Copenhagen group remained there, creating the Nordic Institute for Theoretical Physics (NORDITA), whose first director was Møller, from 1957 until 1971 (Rozental 1985, p. 117).

The Theoretical Study Group in Copenhagen was extremely successful. Each country that was a member of CERN was supposed to send one early career theoretical physicist every year (Iliopoulos 1996). In theory, they would work on theoretical subjects related to the problems posed by the experimentalists, but that original purpose of the group was hardly taken seriously. There was some nuclear physics work, for instance by Aage Bohr and Ben Mottelson, but also some very mathematical work, unrelated to experimental matters, by Rudolf Haag and Gunnar Källén, among others. Møller's own engagement with general relativity meant that he was open to not sticking to CERN experimental concerns. He warmly welcomed, for instance, Haag's idea of studying the Wigner classification of irreducible representations of the Poincaré group (Haag 2010, p. 267).

There were also young physicists associated with the Theoretical Study Group coming from outside the CERN member states, such as Steven Weinberg and Stanley Deser from the USA, who were funded by the National Science Foundation ${ }^{21}$. Deser stayed in Copenhagen for two years, from mid-1955 until the end of the group. Before going to Copenhagen, he was "working, with R. Arnowitt, on the question of renormalization, and non-perturbative solutions of 'non-renormalizable' theories such as $\mathrm{ps}(\mathrm{pv})^{\prime 22}$, that is, (ps=pseudoscalar) meson theories with (pv=pseudovector) derivative couplings, a subject that was related to the work done by Källén and others in the Copenhagen group (Arnowitt and Deser 1955). In Denmark, encouraged by Møller, Deser found a favorable environment to refocus his research on general relativity.

\subsection{Christian Møller and the Chapel Hill project}

DeWitt wrote to Møller in April 1956 telling him about the new research group at Chapel Hill and inviting him to join it as a visiting senior professor in the following academic year ${ }^{23}$. Møller's salary was going to be partially funded by a generous donation from the Research Corporation ${ }^{24}$. That was the very first invitation sent out by DeWitt as director of the Institute of Field Physics. DeWitt hardly knew Møller. They had probably met twice before, the first time at the Tata Institute in India in 1951 and the second time later that same year at the Institute for Theoretical Physics (Bhabha 1951). But Cécile DeWitt-Morette had worked under Møller's supervision in the late 1940s. In a report to the French Department of Education, Møller had the following to say on her stay in Copenhagen:

After having finished her studies in Paris, Madame C. DeWitt, née Morette, spent the academic year 1947-48 at the Institute for Theoretical Physics, Copenhagen, as a Fellow of the Rask-Ørsted Foundation. [...] [Her] work showed her deep understanding of the physical problems and her mastery of the mathematical methods which also is so characteristic of her subsequent work. During

21 Letter from Christian Møller to the National Science Foundation, 16 May 1955. Christian Møller Papers, Niels Bohr Archive, Copenhagen, Correspondence, box 2. Hereafter this collection will be referred to as MP.

22 Letter from Stanley Deser to Christian Møller, 7 April 1955. MP, Correspondence, box 2.

23 Letter from Bryce DeWitt to Christian Møller, 30 April 1956. MP, Correspondence, box 2 .

24 Bahnson Memorandum \#6 (1 November 1956). DF, box Institute of Field Physics. 
her short visit to Copenhagen she made many friends, and due to her amiable personality it was easy for her to get into contact with other physicists at the Institute $^{25}$.

Thus, it is likely that inviting Møller to stay one year at Chapel Hill was her idea. The physics staff there consisted at that time of only six researchers, including Bryce DeWitt, Cécile DeWitt-Morette, Eugen Merzbacher, and Everett Palmatier. In the invitation letter, DeWitt referred to John Wheeler (who was then visiting Copenhagen) and Stanley Deser as those who could explain the project at Chapel Hill and its history. Wheeler, DeWitt stated as his greatest credential, was "to a great extent the godfather of the project" at Chapel Hill. The salary that they offered to Møller was quite attractive, $\$ 9000$ for nine months plus the round trip fare to and from the United States ${ }^{26}$. The letter also mentioned that Eugen Merzbacher was teaching general relativity at Chapel Hill using Møller's textbook. In the same letter, DeWitt further informed Møller that he and Cécile DeWitt were planning "a small conference on gravitational theory" from February 4 to 8, 1957 - which ended up being the 1957 Chapel Hill conference. She added a handwritten postscript to the letter, "You do not know how much your visit would be appreciated", which she signed simply "Cécile". That was as much as Bryce and Cécile DeWitt could do in order to attract Møller to Chapel Hill ${ }^{27}$. He regretted not being able to accept the invitation:

You may know that I am involved in the work with CERN. The Theoretical Study Group will stay in Copenhagen till the fall of 1957, and I have committed myself to leading the Group until that time. Thus, no other obligation can be considered in the year to come. [...] Also the conference in February would have been of much interest to me. However, you have certainly gathered from my above plans that, to my deep regret, I shall also be unable to come on this occasion $^{28}$.

Not having Møller, either as a visitor or at the conference, was for sure a great disappointment to the DeWitts. However it was more than simply a personal disappointment, since Møller would be the representative of the Institute for Theoretical Physics at the conference. And both Bryce and Cécile DeWitt had a clear idea of the relevance of having a well-known physicist from Copenhagen at the event. As the historian John Krige has shown, during the 1950s Copenhagen was perceived by the American funding agencies as the strategic center of the intellectual Cold War in Europe (Krige 2006, p. 169-172). DeWitt then invited to the 1957 conference the three other European physicists from his list, Lichnerowicz, Klein, and Tonnelat. A few months later, as the conference budget was finally confirmed in a favorable manner, that list of European physicists grew.

The Chapel Hill conference finally took place from 18-23 January 1957 and was a huge success. This helped secure further funds for the Institute of Field Physics, allowing Bryce and Cécile DeWitt to consider the possibility of inviting more physicists to join the staff on a temporary basis. Invitations were sent out to Bertel Laurent and to Felix Pirani.

In summer 1956, DeWitt had visited Copenhagen during a trip to Europe, in order to reassert in person the invitation to Møller, now for the academic year 1957-1958 29 .

\footnotetext{
${ }^{25}$ Letter from Møller to DeWitt-Morette, 22 February 1965. MP, Correspondence, Supplement, box 1 .

${ }^{26}$ For comparison, $\$ 1000$ per month was the combined salary of Bryce and Cécile DeWitt.

27 Letter from Bryce DeWitt to Christian Møller, 30 April 1956. MP, Correspondence, box 2 .

${ }^{28}$ Letter from Christian Møller to Bryce DeWitt, 16 May 1956. MP, Correspondence, box 2.

29 Bahnson Memorandum \#5 (16 October 1956). DF, box Institute of Field Physics.
} 
Møller had hesitated several months to confirm the acceptation. In February 1957, after the big conference, he finally answered that he wouldn't be able to stay for nine months at Chapel Hill, since he had already arranged a long stay at Pittsburgh ${ }^{30}$. DeWitt then got impatient and Cécile had to intervene ${ }^{31}$. She insisted that Møller should visit Chapel Hill as soon as his duties with CERN were finished, even if for just a short period:

As we have not been able to "scare" $(! . . .)^{32}$ you into an extended period we would be very happy to have you on the faculty here for a two month period. [...] I hope there will be no unforeseen difficulty preventing you from coming to Chapel Hill ${ }^{33}$.

Møller accepted this last invitation and arranged to stay at Chapel Hill for two months. Apparently, the visit to Pittsburgh had indeed been planned for a long time. In an interview given to the Danish newspaper Berlingske Aftenavis, Møller stated:

That is an old invitation, which I now see myself in a position to accept [...]. The director of the [Carnegie] Institute [of Technology] has so to say repeated the invitation each year, but I have so far been compelled to refuse, among other things because of my work as head of the theoretical division of CERN, which, however, as of next autumn will no longer be located at Niels Bohr's institute. My lectures [in Pittsburgh] will focus on the general theory of relativity ${ }^{34}$.

After his stay at the Carnegie Institute of Technology and a quick visit to Princeton, Møller finally went to Chapel Hill, however for just one month, beginning 25 January 1958. Despite the certain amount of frustration it brought to the DeWitts, having to wait so long for such a short visit, the attempt to get Møller to their institute for a longer period did strengthen the bonds between Copenhagen and Chapel Hill, and thereby made possible the 1957 meeting in Copenhagen.

\subsection{Planning the 1957 meeting in Copenhagen}

DeWitt had also made some efforts to attract to Chapel Hill Stanley Deser and Charles W. Misner as research assistants. Bahnson reported in one of his memoranda:

On the way back from their stimulating summer in [the 1956] Les Houches [Summer School], Bryce DeWitt made a trip to Copenhagen and contacted Dr. Møller and Dr. Deser. It seems likely that Dr. Møller who is an outstanding European physicist, will join the project at Chapel Hill for several months next year and that Dr. Deser may come to Chapel Hill as a research assistant.

30 Letter from Bryce DeWitt to Christian Møller, 12 February 1957. Letter from Christian Møller to Bryce DeWitt, 18 February 1957. MP, Correspondence, Supplement, box 1.

31 Letter from Bryce DeWitt to Christian Møller, 12 February 1957. Letter from Christian Møller to Bryce DeWitt, 18 February 1957. MP, Correspondence, Supplement, box 1.

${ }^{32}$ Footnote in the letter: "Mille excuses - this shows only how much we wanted you for a long stay". She is referring to Bryce DeWitt's harsh letter from 12 February, insisting that Møller should stay for an extended period. This insistence was related to funding restrictions, as she explained to Møller in the letter from 22 February: The grant from the Research Corporation had been awarded on the supposition that Møller would stay at Chapel Hill for an extended period and could not be used for a shorter stay.

${ }^{33}$ Letter from Cécile DeWitt-Morette to Christian Møller, 22 February 1957. MP, Correspondence, Supplement, box 1.

34 "Professor Chr. Møller til USA", Berlingske Aftenavis, 20 August 1957. Danish Newspaper Clippings Collection, volume 34, Niels Bohr Archive, Copenhagen. 
These are very outstanding people in the field of gravitation and contacting them personally was a worthwhile move in the next important steps of trying to attract the best personnel to Chapel Hill now that the problem of attack is becoming more well defined ${ }^{35}$.

However, five months later, the situation had changed. Møller had already made clear that, due to his agreement with Pittsburgh, he could only consider a short visit to Chapel Hill; Deser had received a better offer from Julian Schwinger at Harvard; and Misner had to decline DeWitt's invitation because he needed a place where he could interact with mathematicians - something that was not possible at Chapel Hill. So DeWitt wrote to Bahnson:

Seeing that it was unlikely that Deser and Misner could come to work with us next year and at the same time feeling very strongly that they have very interesting things to say and that we could do some very interesting things together, I got in touch with them to find out if they would be interested in getting together in Europe this summer. As you know, Deser is currently in Europe anyway and Misner was going there just for a vacation. Both of them responded very favorably and we also got in touch with Professor Schwinger of Harvard to find out if he would be interested in joining the group. He too, responded favorably since he was anyway going to be in Europe. According to present plans, a group of six of us will be in Copenhagen during the month of July working at the Institute for Theoretical Physics directed by Bohr. The six included myself, Deser, Misner, Laurent, Schwinger and Professor Klein of Stockholm ${ }^{36}$.

We do not know the reason why Schwinger had to cancel his participation. It is understandable that Møller would not have been expected to join the meeting - even though he did ultimately join it - because the Theoretical Study Group was about to be dissolved, NORDITA was being created, and, in addition to all that, Møller was about to move to the United States for about half a year.

In any case, organizing such a meeting required a certain amount of funding. DeWitt applied to both the National Science Foundation and the US Air Force. As we already know, it was the latter who ended up funding the meeting. As Bahnson wrote in a memorandum:

Bryce is leaving on MATS Air Force transportation [Military Air Transport Service] the end of May or the first of June [1957] to attend the conference of mathematics in Lille, France from June 3 to 8 . He will spend the month of July in Copenhagen with five other outstanding physicists who will try to get a fresh point of view on the problem [of the quantization of gravity] in associating with one another, and I believe the next year will show exciting progress in the solution of a most profound and difficult problem ${ }^{37}$.

Bahnson, a philanthropist with no formal training in physics, sometimes had rather fanciful expectations. In this case - that the following year was to "show exciting progress" in quantum gravity - they were not altogether unfounded. We will return to this question in the conclusions.

35 Bahnson Memorandum \#5, see footnote 29.

${ }^{36}$ Memorandum from Bryce S. DeWitt to Agnew H. Bahnson, 19 March 1957. DF, box Institute of Field Physics.

37 Bahnson Memorandum \#9 (7 May 1957). DF, box Institute of Field Physics. 


\section{An analysis of Bryce DeWitt's report}

We now turn to the analysis of Bryce DeWitt's 1957 report. The division of Section 4 follows the report's structure, and the reader might be able to profit from it most by reading this section and the report in parallel.

\subsection{The theory of measurement}

The first section of the report discusses the measurement of a quantum gravitational field. It is remarkable that DeWitt decided to begin with such an epistemological issue. Since Møller took part in these discussions and since he joined the meeting only during the last eleven days, we can deduce that the presentation of the report did not follow the order of the discussions. Therefore, putting the measurement problem at the beginning was a choice of DeWitt's.

In the late 1960s, DeWitt would become the most well known supporter of Hugh Everett's interpretation to quantum mechanics, but in 1957 he was still quite close to Niels Bohr's views ${ }^{38}$. Indeed, the definition that DeWitt provides of a measurement is entirely Bohrian: measurements are consistency problems (Kalckar 1971, p. 127). He began the report with the claim that in the development of physical theories that extend previous concepts, at some point one must examine the self-consistency of the entire new framework - i.e., at some point one must develop an analysis of the possible measurements within that theory. DeWitt often started his texts with general methodological lessons learned from historical episodes. In this case he was referring to the early history of "electrodynamic theory", i.e., quantum electrodynamics, where the examination of the measurement problem in quantum theories came after the development of the mathematical formalism. Before returning to the 1957 report, we shall briefly discuss this historical episode, as it is essential for understanding DeWitt's position.

In 1929, Werner Heisenberg wrote a letter to Niels Bohr explaining some ideas that he had just sketched about the measurement of electromagnetic fields in quantum theory. In close analogy with the position-momentum uncertainty relations, Heisenberg expected that the electromagnetic field components - which were also non-commuting variables, according to the quantum electrodynamical formalism that he had just developed with Wolfgang Pauli (Heisenberg and Pauli 1929) - would be subjected to similar uncertainty relations. Heisenberg then designed two thought experiments with the purpose of visualizing the limitations on the simultaneous measurement of field components. These were just preliminary investigations, as he explained to Bohr:

I believe that the essential features here are correct, but the utilization of the average values of $\mathrm{E}$ [the electric field] and $\mathrm{H}$ [the magnetic field] in the cube is still not quite solid. But would you in principle consider such a discussion reasonable ${ }^{39}$

That letter was discussed in Copenhagen and two young physicists - Lev Landau and Rudolf Peierls - decided to tackle the problem themselves. There was at the time a widespread belief that the quantum theory of relativistic systems - in particular, quantum electrodynamics - was essentially wrong, due to the occurrence of several divergencies in the calculations (whose solution would appear only in the late 1940s

\footnotetext{
38 This shift in DeWitt's opinion on the foundations of quantum mechanics - from a Bohrian perspective to a radical Everettian one - will be analyzed in a forthcoming article by one of the authors $(\mathrm{TH})$.

${ }^{39}$ Letter from Werner Heisenberg to Niels Bohr, 16 June 1929. Translated in (Kalckar 1996, p. 7).
} 
with the full development of renormalization methods). Landau and Peierls decided to search for the source of all the troubles. Following Bohr's ideas - "in the description of atomic phenomena, the quantum postulate presents us with the task of developing a 'complementarity' theory the consistency of which can be judged only by weighing the possibilities of definition and observation" (Bohr 1928, p. 580) - they decided to use a variant of Heisenberg's thought experiment to show that the very idea of a measurement was meaningless in relativistic quantum theory.

Their argument goes as follows. In order to measure an electromagnetic field one must observe its action on a test charge, whose movement provides all information one can obtain about the field. Landau and Peierls chose a point electron as the test charge. They demonstrated, through a thought experiment, that in a quantum context the radiation reaction of the test charge is uncontrollable, leading to the following fundamental uncertainty for each component of the electric field:

$$
\Delta E_{i} \gtrsim \frac{\sqrt{\hbar c}}{(c T)^{2}} .
$$

Since the mathematical formalism does not predict such single component uncertainties (only pair uncertainties), Landau and Peierls concluded - following Bohr's terminology - that the quantum theory of the electromagnetic field, in particular the relation between definition and observation, is not consistent. Generalizing that reasoning, they claimed that "in the correct relativistic quantum theory (which does not yet exist), there will therefore be no physical quantity and no measurement in the sense of wave mechanics" (Landau and Peierls 1931, p. 69, translation in ter Haar 1965, p. 50).

Niels Bohr was deeply bothered by these conclusions (Darrigol 1991; Jacobsen 2011). It took him almost three years to work out an adequate refutation, which came in the form of a long, difficult, and subtle paper written with Léon Rosenfeld (Bohr and Rosenfeld 1933). Its message, however, was quite simple: In order to make the best possible measurement, one should use not an electron, but a massive test charge (with no fixed charge/mass ratio), with macroscopic dimensions (so that its charge density is finite), and attach it to a series of springs (which act as a compensation device). Then the radiation reaction may be entirely eliminated. The resulting uncertainties for the electromagnetic field obtained from a thus improved thought experiment are exactly those expected from the mathematical formalism of quantum electrodynamics. Therefore, quantum theory may be consistently applied to relativistic systems after all, in the same way that it was applied to non-relativistic systems.

We now return to the 1957 report. DeWitt explains that the situation in the quantum theory of the gravitational field is different, since "the question of "measurability' has been raised even before the advent of a formalism". The first discussions about the measurability of the quantum gravitational field dates back to the mid1930 (Bronstein 1936; Solomon 1938; see also Blum and Rickles 2017; Hartz and Freire 2015, and references therein $)^{40}$. However, DeWitt is not referring to those early efforts, of which apparently he was not aware, but actually to the 1957 Chapel Hill conference, where the measurement of a quantum gravitational field was discussed several times.

The Chapel Hill conference had two proceedings: One edited by Bryce DeWitt was published in the Reviews of Modern Physics in July 1957, and another written by Cécile DeWitt-Morette circulated as an internal report to the US Air Force in the same style as the Copenhagen report, but considerably larger (167 pages). The latter proceedings were produced between the end of January and mid-March

\footnotetext{
40 These discussions were still ongoing some fifty years later (von Borzeszkowski and Treder 1988, Chapter 3).
} 
1957. Most of the talks and discussions were paraphrased, but several large sections of the discussions were reproduced verbatim (in quotation marks). This was possible due to major advances in portable tape recording technology in the 1950s, allowing Cécile DeWitt-Morette to go around during the conference and record virtually all the discussions ${ }^{41}$. Of course, the poor quality of the recording demanded complementary methods. As she explained:

It can hardly be said that the report [of the Chapel Hill conference] gives a perfectly true picture of the conference. The report has been prepared from notes taken during the session, from material given by the authors, and from tape recordings. (The reporters have hoped to have a stenographic transcript available, but the cost of this transcript was beyond common sense.) Some contributions have been very appreciably abridged, some are reproduced practically verbatim, some are extended, and some have not been recorded, depending largely on the "communication" (both material and intellectual) between authors on the one hand and reporters and editors on the other (DeWitt and Rickles 2011, p. 34).

Due to these limitations, it cannot be deduced from the proceedings who was (were) the one(s) to raise the problem of measurement of the gravitational field in discussion. The proceedings indicate - in Cécile DeWitt-Morette's own words - that, after Peter Bergmann's talk, which initiated the second half of the conference, dealing with quantum gravity, the following discussion took place:

Discussion then turned to the problems of measurement of the gravitational field. This item was placed first on the agenda in an attempt to keep physical concepts as much as possible in the foreground in a subject which can otherwise be quickly flooded by masses of detail and which suffers from lack of experimental guideposts. The question was asked: What are the limitations imposed by the quantum theory on the measurements of space-time distances and curvature? Since the curvature is supposed to be affected by the presence of gravitating matter, an equivalent question is to ask: What are the quantum limitations imposed on the measurement of the gravitational mass of a material body, and, in particular, can the principle of equivalence be extended to elementary particles? (DeWitt and Rickles 2011, p. 167)

Probably that was the first time that the 1950s generation expressed a concern about the measurement of a quantum gravitational field and the fact that it was placed at the beginning of the quantum gravity session, just as it was placed at the beginning of the Copenhagen report, makes it highly probably that it was Bryce DeWitt who put this problem on the agenda. In the proceedings, he appears, however, only as the (probable) author of an editorial note.

The main talk on measurements at Chapel Hill was given by Helmut Salecker, who presented his work with Eugene Wigner. He began by demonstrating that one can measure space-time distances using only a clock, with no need for rods. All quantum limitations in those measurements are therefore a consequence of the quantum limitations of clocks. Wigner had always been puzzled by Bohr and Rosenfeld's reasoning because the measurement device they proposed could hardly be reproduced in a real experiment (Wigner 2002, p. 9). According to Bohr, thought experiments should not be limited by what is actually possible; it should only be restricted by the rules of the formalism applied to idealized experimental situations. The tension between Bohr-Rosenfeld-type ideal clocks (with which the uncertainties implied by the formalism could be reproduced exactly) and physically feasible clocks (with which these

\footnotetext{
${ }^{41}$ Information obtained in a conversation of one of the authors (TH) with Cécile DeWittMorette, 4 August 2011, Austin, TX, USA.
} 
uncertainties could only be approximately reproduced) was Wigner's main concern in his work with Salecker (Salecker and Wigner 1958).

After Salecker's talk at Chapel Hill, Rosenfeld took the floor to comment on the difficulties in measuring the quantum gravitational field. In the electromagnetic case discussed by him and Bohr in 1933, it had been necessary to use both positive and negative charges, in order to reduce the electromagnetic field of the test charge to a dipole field (with no monopole term). It had also been essential for the 1933 argument to be able to arbitrarily adjust the charge to mass ratio. In the gravitational case, the situation was quite different on both accounts. Rosenfeld thus agreed with Salecker's claim that the measurement of the gravitational field has further limitations than in the electromagnetic case, and probably would even produce single-component uncertainties, in the manner of Landau and Peierls (DeWitt and Rickles 2011, p. 178-179). The implications of such single-component uncertainties would have been dramatic: In essence, they would have made any straightforward quantization of the gravitational field impossible. At this point, Wheeler intervened. In Cécile DeWitt-Morette's words:

Wheeler suggested that perhaps one should simply forget about the measurement problem and proceed with other aspects of theory. The history of [quantum] electrodynamics shows that it is always a ticklish business to conclude too early that there are certain limitations on a measurement. He would propose rather to emphasize the organic unity of nature, to develop the theory (i.e., quantum gravidynamics) first and then to return later to the measurement problem. He suggested that this was particularly appropriate when we don't even understand too much about the classical measurement process! (DeWitt and Rickles 2011, p. 179)

We see here the main antagonism of quantum gravity in 1957, that between Wheeler and DeWitt: The latter meticulous and diligent, trying to get the foundations straight, the former sanguine and visionary, interested only in the exciting things one might be able to do with quantum gravity. We will see this antagonism pop up throughout the report. At Chapel Hill, in any case, the measurement issue was not entirely forgotten. In particular, Rosenfeld expressed some second thoughts. He stated that "perhaps his original pessimism in regard to the measurability of gravitational fields, as compared to the electromagnetic case, might be unjustified" (DeWitt and Rickles 2011, p. 184185). He argued that perhaps the uncertainties in the position and momentum of the test charge (i.e., in the gravitational case, a test mass) influence different components of the gravitational potential, so that any single component could be measured with arbitrary precision.

Despite Rosenfeld's cautious optimism, the discussions at Chapel Hill led DeWitt to state - in the Copenhagen report - that measurement issues in the quantum theory of the gravitational field "have been mainly directed toward proving the inapplicability of quantum mechanics in the gravitational domain". He then goes on, surprisingly at first glance, to suggest that perhaps the expectation of obtaining strong evidence against the quantum gravity program from measurement analyses may just be wishful thinking, because of the complexity of the problem. DeWitt thus seems to be subscribing here to Wheeler's opinion that one should not approach the problem of measurements in quantum gravity for the time being. We thus have here an interesting historical problem: Why then did DeWitt dedicate so much attention in Copenhagen to measurement matters? There are two possible answers.

First, DeWitt did not have at the time a good argument for the quantization of the gravitational field, the problem to which he was dedicating his career. His basic argument in 1957 was the following:

No apology will be made for [the desire to attack the problem of developing a quantum theory of gravitation], although needless to say, recent experiments 
have nothing to do with it! In the author's opinion it is sufficient that the problem is there, like the alpinist's mountain (DeWitt 1957b, p. 377).

At the same time, there were theoretical arguments on measurability supporting the idea that perhaps the gravitational field should not be quantized. We could mention Salecker's and Rosenfeld's measurement arguments, as well as some other thought experiments formulated at the Chapel Hill conference that raised some doubts concerning the necessity of quantizing the gravitational field (DeWitt and Rickles 2011, p. 247-260; see also Zeh 2011). Thus, in order to protect his research project, DeWitt had to be able to argue against such doubts. That is the purpose, for instance, of the long calculation that DeWitt presented in the Copenhagen report. The great importance that he attributed to such a calculation can be seen from the fact that he had already included it in the Chapel Hill report, during the editing process (DeWitt and Rickles 2011, p. 167-169). As opposed to Wheeler, DeWitt did not want to (or did not feel able to) cavalierly brush such objections aside.

Second, DeWitt perhaps had some cautious hopes that the measurement analysis could be of some aid in the development of a quantum theory of gravitation. We will come back to this point in a few paragraphs.

DeWitt mentions that the discussions about measurement were raised by Christian Møller, who had discussed the matter with Wigner. Møller had also been interested in understanding the behavior of a clock in a gravitational field. It was well known (Møller 1952, p. 247) that the proper time $\tau$ of an observer moving with velocity $v$ in a region subjected to a gravitational field with the scalar potential $\chi$ is given, in a system of coordinates that is time-orthogonal, by the expression

$$
d \tau=d t \sqrt{1+2 \chi / c^{2}-v^{2} / c^{2}}
$$

where $t$ is the proper time of an observer at rest in the absence of a gravitational field. Møller wanted to deduce expression (2) from a clock model - also for pedagogical reasons, as discussed in Section 2.2 - and to evaluate what would be the changes in expression (2) if one considered a real (Wignerian) clock (not an ideal Bohrian one).

Møller also expressed some concerns about approaching measurements at that stage of the development of the quantum theory of the gravitational field, since Bohr and Rosenfeld in 1933 had to systematically use the mathematical formalism of quantum electrodynamics in order to find the best possible way of measuring the electromagnetic field - as DeWitt mentions in the report:

Professor Møller quoted a statement made by Professor Bohr after finishing his famous "measurement" paper with L. Rosenfeld, viz. "During the course of our study of the quantum limitations on the measurability of the electromagnetic field we made every possible mistake. In each case, in order to extricate ourselves, we had to go back and look at the formalism!"

That was meant to substantiate Wheeler's opinion that the measurement problem in quantum gravity was to be left alone for the time being. Instead of following Møller's advice, however, DeWitt ultimately decided to invert the argument and to attempt and define the formalism (i.e., the commutation relations of the theory) in terms of the measurement analysis. In this manner, the above quotation came to be the main source of inspiration for DeWitt's work in the five years following the Copenhagen meeting (Hartz and Freire 2015). In his contribution to the famous collection Gravitation, edited by Louis Witten in 1962, DeWitt stated:

The author [DeWitt] is heavily indebted [to Bohr and Rosenfeld's 1933 paper] as will be immediately apparent in the sections to follow. This indebtedness may seem in one respect surprising, not, to be sure, because of any present-day 
diminution in the importance of this classic work, but because its content, as Bohr and Rosenfeld have themselves repeatedly indicated, was guided in every way by the existence of an already developed formalism, whereas here we are trying to "put the cart before the horse" - to develop the formalism itself with the aid of the ideas of the theory of measurability (DeWitt, 1962, p. 270).

This way of defining the mathematical formalism from the measurement analysis was central in much of DeWitt's later work on quantum field theory and quantum gravity (see, e.g., DeWitt 1971, 2003, p. 30-144; see also DeWitt 2011, p. 15).

\subsection{Topological problems}

This section at first glance appears somewhat out of place in this workshop on quantum gravity. DeWitt does offer a motivation for the study of topological problems in the context, stating that the classical behavior of the gravitational field is "not well understood" and implying (though only by historical analogy) that this is a necessary prerequisite for the construction of the corresponding quantum theory. There are, however, much more important reasons for the prominent place of a section on topology in this report and that is the role played by topological considerations in the research program of John Archibald Wheeler.

In 1953, after a decade of pursuing a program of reducing all of physics to direct particle interactions (as in Wheeler-Feynman electrodynamics), Wheeler had switched gears entirely. In the academic year 1952/53, he was teaching his famous first course on relativity, both special and general, with the aim of learning general relativity himself in order to, it appears from his notebooks, also cast the gravitational interaction in the form of a direct particle interaction. In the course of the term, however, Wheeler became enamored with Einstein's unified field theory program. This was, in a rather explicit sense, the exact opposite of Wheeler's program, namely to reduce everything to fields, as outlined by Einstein in the appendix to the fourth edition of his "Meaning of Relativity", which found an interested reader in Wheeler in late March $1953^{42}$ :

There is $[\ldots]$ the conviction that one cannot keep side by side the concepts of field and particles as elements of the physical description. [...] The field concept, however, seems inevitable, since it would be impossible to formulate general relativity without it. [...]

For this reason I see in the present situation no possible way other than a pure field theory, which then however has before it the gigantic task of deriving the atomic character of energy. (Einstein 1953, p. 164-165)

How now to face this "gigantic task?" Wheeler at first focused on studying solutions of the vacuum Einstein-Maxwell equations with localized energy, the so-called geons (Wheeler 1955), but soon turned toward an approach that had first been suggested (though not pursued much further) by Einstein himself, already in the mid-1930s (Einstein and Rosen 1935), namely to model particles in a pure field theory through the topology of space(-time). Wheeler's paradigmatic example of topology as particles was the wormhole, a topological handle with closed electric field lines partially trapped inside, such that field lines appear to be diverging from the two points where the handle is attached to the rest of space (mimicking, in Wheeler's words, charge without charge).

${ }^{42}$ The above reconstruction of Wheeler's path is the preliminary result of a detailed study of Wheeler's notebooks and his intellectual trajectory in the 1950s, currently being pursued by one of the authors $(\mathrm{AB})$ together with Dieter Brill. 
While clearly inspired by Einstein, Wheeler's program differed from Einstein's in one central aspect. Einstein had hoped that the particle-like entities obtained in a unified field theory would (approximately) obey the laws of quantum mechanics, without these laws (with all their conceptual baggage, to which Einstein objected) having to be explicitly introduced into the foundations of the theory. For Wheeler, on the other hand, his classical considerations were merely a prelude to the eventual construction of the full quantum theory. This allowed him to bracket the obvious shortcomings of the classical topology as particles program, e.g., the fact that one could deduce an upper limit on the charge-to-mass ratio that was many orders of magnitude too small to reproduce even the most massive of elementary particles (Misner and Wheeler 1957). Wheeler (1957) was rather vague on how exactly these shortcomings of the program were to be cured in the quantum theory, but it centrally involved Feynman path integral quantization with integration over field configurations with non-trivial topologies and a special emphasis on the quantum fluctuations of the metric at the Planck scale. Topology was thus an essential element of Wheeler's approach to quantum gravity or, more properly, quantum gravity was an essential element of Wheeler's particles-from-topology program.

DeWitt did not mention these speculations of Wheeler's, instead framing the section on topology in a more conservative manner merely as an exploration of the classical theory. We will see in more detail in Section 4.5 that DeWitt was both highly skeptical of Wheeler's program and hesitant to criticize it outrightly, which explains his cautious formulation. This caution also most likely coincided with that of the representative of the Wheeler school in Copenhagen, Misner. He had worked both on the formal questions of the Feynman quantization of general relativity (see the next section) and had co-authored with Wheeler the paper on the classical theory of wormholes; but the highly speculative paper in which Wheeler sought to connect the two had been written by Wheeler alone. The discussion of topology at the workshop therefore appears to have focused entirely on classical issues, with Wheeler's "quantum foam" speculations looming as a distant motivation in the background.

One aspect discussed in Copenhagen was whether one might also be able to describe the sources of a scalar (Yukawa) meson field (believed at the time to be the adequate description of the interaction between nucleons) using wormhole-like structures ("topological tricks" as they are referred to in the report) ${ }^{43}$. In the Einsteinian tradition, Wheeler had focused exclusively on the gravitational and electromagnetic interactions. Indeed, he was highly skeptical of the meson theories of nuclear interactions of the time, as he stated, e.g., his 1954 Richtmyer lecture ${ }^{44}$ :

I find it impossible to regard the meson field hypothesis as more than a free invention - ingenious and suggestive, to be sure, but only a free invention -

\footnotetext{
${ }^{43}$ The report mentions that this problem was studied with the help of Arthur Komar who was a postdoc in Copenhagen at the time, apparently studying "the interaction of scalar and gravitational fields". There is no trace of this work in Komar's publications, but we do find the following remark in Wheeler's letter of reference for Komar to Aage Bohr in Copenhagen (24 April 1956, Wheeler papers, Box 15, Komar correspondence): "Komar has a strong background in mathematics, especially differential geometry [...] His background in nuclear physics and quantum electrodynamics is nowhere near so advanced, but I know he is particularly eager to do something in field theory and wants to come to Copenhagen for that reason". Komar does not appear to have pursued this any further after moving to Bergmann in Syracuse the following year.

${ }^{44}$ In contrast, Wheeler very much believed that the neutrino would remain an integral part of fundamental physical theory, despite the fact that it had not even been discovered at the time of the 1954 lecture. His attempts to construct wormholes that acted as sources of a classical neutrino Weyl spinor field failed, however (Klauder and Wheeler 1957).
} 
that distracts us from following out the implications of the theories we already have $\mathrm{h}^{45}$.

But for someone like Deser, coming from a high-energy nuclear physics background, it was a natural question to inquire whether Wheeler's methods could also be employed for the state-of-the-art theories of the nuclear interactions. As DeWitt writes, they found that this worked just fine for a massless ${ }^{46}$ meson field, while there were some difficulties with a massive field ${ }^{47}$.

While we have no details of these calculations, the difficulty is quite easily understood by looking at Misner and Wheeler's treatment of electromagnetic wormholes. Their study had consisted of two steps: First, ignoring the dynamics of the metric, they had studied the vacuum Maxwell equations in a doubly connected space ${ }^{48}$ and verified the possible existence of wormholes. This study did not involve any reference to the Einstein equations or the dynamics of general relativity and worked just as well for the (vacuum) Yukawa (massive Klein-Gordon) field equations.

The second step in Misner and Wheeler's "Classical Physics as Geometry" was now to take into account also the dynamics of the metric. After having established the possibility of constructing charges from topology, they had then disregarded the topological aspects, treating a wormhole merely as two point charges, thereby neglecting the metric within the actual wormhole. Now, there was no exact solution of the Einstein equations for more than one massive particle, but, as Misner recalled:

All through this time with Wheeler, I was pushing the idea that you could use the initial value problem in general relativity to get some results that were too complicated to get dynamically ${ }^{49}$.

Misner had studied the work of André Lichnerowicz (1944) who had shown how to solve the initial value equations ${ }^{50}$ for the metric of $n$ massive bodies. For the joint paper with Wheeler, Misner had then generalized Lichnerowicz's solution of the initial

45 John Archibald Wheeler papers, American Philosophical Society, Philadelphia, Box 182. In the remainder of the document, this collection will be referred to as JAWP.

${ }^{46}$ Of course the classical scalar field equation has no mass associated with it, only a characteristic length scale, which in the quantized field theory becomes the Compton wavelength of the field quantum. It has however, as witnessed by the report, been common for decades to call the classical field equation "massive" as well, and we do not wish to be particular.

${ }^{47}$ Tellingly, given Wheeler's objections to meson theory, when Misner reported back to Wheeler on his attempts to incorporate mesons in the their program, he did not mention the possible physical relevance, but only the calculational simplicity: "The scalar photon [i.e., the massless meson] makes a good example of how the process works, without nearly as much work as for electromagnetism". (Letter from Misner to Wheeler, 10 August 1957, JAWP, Box 18, Misner Correspondence)

${ }^{48}$ We use here Wheeler's terminology, where a "doubly connected space" is a space "with a handle", where two given points are "doubly connected" because there are two connections from one point to another, one through the bulk of the space and one through the handle. This is not to be confused with another possible usage of the term "doubly connected", implying a fundamental group of order 2. Spaces that are doubly connected in Wheeler's sense have an infinite fundamental group and would thus be categorized as infinitely connected in this latter terminology. A detailed historical study of the origins of this incompatible terminology is beyond the scope of this paper.

49 Interview by Dean Rickles and Don Salisbury, 16 March 2011, Niels Bohr Library and Archives, American Institute of Physics, College Park, MD.

${ }^{50}$ In the report these are referred to as the Fourès equations, after Lichnerowicz's student Yvonne Fourès-Bruhat (later Yvonne Choquet-Bruhat) who had rigorously proven the wellposedness of the initial value problem in general relativity (Fourès-Bruhat 1952). See also (Lichnerowicz 1992). 
to the case of $n$ charged particles (wormhole mouths). Such a generalization was only possible for particles with a $1 / r$ Coulomb potential, for which there existed an exact solution in the one-particle case, and thus could not be performed for a massive scalar field, with its exponentially decreasing Yukawa potential. This appears to have been the problem that Deser and Misner encountered in Copenhagen. It was, however, not a problem of principle, but one of solving the Einstein (or at least the initial value) equations for a particle creating a non-Coulomb potential.

It should be noted that the $n$-body (charged or uncharged) initial value solution not only makes no use of a wormhole topology, it also does not involve any singularities of the metric (as opposed to the Schwarzschild or Reissner-Nordström solutions) in order to ensure the mathematical well-posedness of the initial value problem. For this reason, in his adaptation of the Reissner-Nordström solution to an $n$-body solution of the initial value equations, Misner had first formulated the Reissner-Nordström metric in isotropic coordinates ${ }^{51}$. In these coordinates the singularity at the horizon occurs only in the time-time component $g_{00}$ of the metric (i.e., in the lapse). Since $g_{00}$ is unconstrained by the initial value conditions, the singularity could then be simply be smoothed over in the initial value reformulation, and also in its generalization to $n$ bodies. The spatial Reissner-Nordström metric $d l^{2}$ in isotropic coordinates reads ${ }^{52}$ :

$$
d l^{2}=\left[(1+m / 2 r)^{2}-(q / 2 r)^{2}\right]^{2}\left(d r^{2}+r^{2} d \Omega\right) .
$$

Here, the mass $m$ and the charge $q$ are given in "natural units", where both Newton's constant and the speed of light are set equal to 1. For the case of several point charges, Misner replaced the spherical coordinates in the round brackets by Cartesian ones (the many-body problem is no longer spherically symmetric) and the conformal factor in square brackets by the more general expression $\left[\chi^{2}-\phi^{2}\right]^{2}$, with $\chi$ a generalization of the Schwarzschild part

$$
\chi=1+\sum_{a} \frac{\alpha_{a}}{\left|\mathbf{r}-\mathbf{r}_{a}\right|}
$$

and $\phi$ a generalization of the Coulomb part

$$
\phi=\sum_{a} \frac{\beta_{a}}{\left|\mathbf{r}-\mathbf{r}_{a}\right|}
$$

where the sums go over all the charged point particles (wormhole mouths) located at the points with space coordinates $\mathbf{r}_{a}$ and the constants $\alpha_{a}$ and $\beta_{a}$ are directly related to the masses and charges of those particles, albeit in a non-trivial manner. The topology of the initial three-dimensional hypersurface is then simply Euclidean with the set of points $\mathbf{r}_{a}$ removed.

In their paper, Wheeler and Misner had not commented on this fact. But the report gives some indication as to the reasons for the adaptation of the simplified topology: Apparently Misner had tried to construct a metric for an actual wormhole topology, already early on in his thesis work ${ }^{53}$, but had failed. Revisiting this problem in Copenhagen, he finally succeeded. As he reported to Wheeler after the meeting:

I looked again at the situation of a wormhole biting its tail $\left(S^{2} \times S^{1}\right.$, a torusy thing in 3-dim.) which was the first thing I did for you several years ago. Then

\footnotetext{
${ }_{51}$ Misner refers to these coordinates in the report as conformal coordinates, as the conformal flatness of the spatial Reissner-Nordström metric is manifest in these coordinates.

${ }^{52}$ There is a exponent of 2 missing on the square brackets in equation 243 of (Misner and Wheeler 1957).

${ }^{53}$ He had been working on topological issues as early as 1955, as witnessed by the abstract of his presentation at the APS meeting in April of that year (Misner 1955).
} 
I found no solution of the Einstein-Maxwell equations was possible, but this time I find them ${ }^{54}$.

Misner would go on to work this out in detail after Copenhagen. His further work on the initial value problem for the wormhole would play an essential role in his contribution to the Arnowitt-Deser-Misner (ADM) collaboration, as we will discuss in Section 4.4. His renewed study of the wormhole initial conditions finally led to his 1960 paper, a two-page affair with no references at all to quantum gravity. We witness here an interesting feature of the renaissance of General Relativity, where a problem that first arises within a far-ranging research scheme, in this case referencing both unified field theory and the quantization of gravity, becomes divorced from these speculations and instead establishes itself as a research topic within the newly emerging field of general relativity proper. We saw in this section how topology divorced itself from the quantum problem, offering as it did interesting problems in its own right. In the next section, the first one to treat the actual quantization of gravity, we will see that the separation was in a sense mutual, with DeWitt trying to push back the Wheelerian speculations and the excessive complications introduced into the problem of quantizing gravity by dragging in topology, arguing instead for an isolation of the specific problem of quantum gravity.

\subsection{Feynman quantization}

Feynman quantization (in its application to field theory at least) was in 1957 still no more than a heuristic tool, far removed from any kind of formal rigor. The results that Feynman had obtained in quantum electrodynamics using his functional integral methods had really only been able to make an impact after Freeman Dyson had managed to re-derive them using more rigorous methods (the mainstream covariant techniques of relativistic quantum field theory $)^{55}$. While drawing inspiration from Feynman's work for this re-derivation, Dyson recalls having strongly criticized Feynman's approach for its lack of rigor:

I argued with him a lot, because I still had strong resistance to his way of doing things [...] I said to him: "Look, you've got to get the mathematics right, otherwise it doesn't make any sense. You've got to have some solid foundation in mathematics. It's alright to draw the pictures, but..." 56

Still, Feynman quantization did not entirely fade from view in the 1950s, both because of its conceptual interest ${ }^{57}$ and the promise it appeared to hold for providing nonperturbative methods in quantum field theory, so sought after in order to get a handle on the strong-coupling nuclear theories of the day.

${ }^{54}$ Letter from Misner to Wheeler, 10 August 1957, JAWP papers, Box 18, Misner Correspondence. The letter is written so long after the conference (and sent from Salzburg) as Misner went on a tour of Europe with his brother after the Copenhagen workshop (Email from Charles Misner to one of the authors (AB) of 1 April 2016).

55 See (Schweber 1994).

${ }^{56}$ Web of Stories interview with Dyson, http://www.webofstories.com/play/freeman. dyson/71.

57 As witnessed by the panegyrics in an early paper by Yoichiro Nambu (1950): "[Feynman's] ingenious method is indeed attractive, [...] because of its way of thinking which seems somewhat strange at first look and resists our minds that are accustomed to causal laws. According to the new standpoint, one looks upon the world in its four-dimensional entirety. A phenomenon that will come into play in this theatre is now laid out beforehand in full detail from immemorial past to ultimate future and one investigates the whole of it at glance". 
As DeWitt outlines in the report, one of the main proponents of Feynman quantization and its application to gravity was John Wheeler, who had of course been Feynman's thesis advisor. He had suggested the Feynman quantization of general relativity as a thesis topic to Misner, indicating that this might be something that might be done within half a year. Misner had jumped at this chance, given that the other option was to write a 700-page thesis on axiomatic quantum field theory with Arthur Wightman ${ }^{58}$. Misner had then, however, soon switched gears and was mainly working on the so-called "already unified theory" (electromagnetism without electromagnetism) (Misner and Wheeler 1957), until it was pointed out to Wheeler and Misner by Bergmann that this theory had largely already been worked out by George Rainich thirty years earlier. In order to present original work for his PhD thesis, Misner had returned to the work on Feynman quantization in early 1957 and had, indeed within a few months, written a short thesis on the matter, which was published in the special issue of Reviews of Modern Physics dedicated to the Chapel Hill conference, despite the fact that Misner had not presented this work of his there ${ }^{59}$.

The aspect of Feynman quantization mainly discussed in Copenhagen, however, had been done early on by Misner, before getting distracted by the already unified theory $^{60}$. Wheeler had selected Misner for the problem of Feynman quantization because of his mathematical prowess, so it was an obvious candidate for the first problem to tackle: The measure involved in the functional integration over metric configurations. In his thesis work, Feynman had only treated integration over actual paths, i.e., over the time-dependent position of a quantum-mechanical particle. There were two ways to extend this to field theory: The first was to Fourier decompose the field and then treat each Fourier mode as a quantum mechanical oscillator to be treated according to Feynman's original quantum mechanical rules. This was what Feynman (1950) himself had done when he first applied his methods to QED proper. However, as Misner pointed out in his thesis, this again requires resorting to perturbations around a flat metric, since only then does one have a complete set of solutions (to the linearized vacuum Einstein equations) to do Fourier analysis with.

Wheeler had thus suggested a different way to do the functional integration for the case of gravity, namely to do spatial (rather than $k$-space, as in the Fourier decomposition approach) discretization and then integrate over the field values at the discrete space points $x_{i}$ in the usual, quantum mechanical, Feynman manner. The most straightforward way to do this was simply to take as measure the product over all $d g_{\mu \nu}\left(x_{i}\right)$ and then integrate in a straight line from the values of the metric at the beginning of an infinitesimal time interval to their values at the end ${ }^{61}$. But as Misner had already pointed out in his thesis, the measure made no distinction between metrics with different signatures and thus there was no indication how it should return the Minkowski metric as the classical limit in the vacuum case. Misner had thus proposed a measure that also contained an inverse power of the negative metric determinant $(-g)$, which would become singular for processes in which the metric tried to change its signature (i.e, went through a point where the metric determinant was zero), concluding that

The range of integration $[. .$.$] is the connected region of g_{\mu \nu}$-space containing $g_{\mu \nu}=\operatorname{diag}(-1,1,1,1)$ and bounded by $\operatorname{det} g_{\mu \nu}=0$.

\footnotetext{
58 See interview of Misner by Chris Smeenk, 22 May 2001, https://www.aip.org/ history-programs/niels-bohr-library/oral-histories/33697.

59 On this story see (Wheeler and Ford 2000, p. 267-268) and interview with Misner mentioned in footnote 49.

60 See (Misner 1957, p. 497).

61 The same infinitesimal segmentation of the total time interval had been done by Feynman for quantum mechanics.
} 
Misner had identified $(-g)^{-5 / 2}$ as the correct power of the negative metric determinant to appear in the measure, using his notion of homogeneity group - in essence demanding that the measure for the integration over the metric variables at some point $x_{i}$ be invariant under the group of coordinate transformations at that point ${ }^{62}$. His argument had involved no recourse to the actual Lagrangian determining the dynamics of the metric, a feature he had explicitly highlighted in his thesis. From DeWitt's report we can gather that Laurent in Copenhagen had managed to derive Misner's measure from a different line of argumentation, based on the explicit structure of the Lagrangian; but no details are given and Laurent never published on the matter ${ }^{63}$. Misner's measure is obviously not the only measure to become singular for a change of signature, and DeWitt had apparently constructed a "preliminary measure" which would also be singular in case the initial or final hypersurface of the transition amplitude being calculated ceased to be space-like; he would eventually come to dismiss Misner's measure and the entire notion that the measure could be directly determined from the invariance group altogether (DeWitt 1962b) ${ }^{64}$.

The main focus of the discussions in Copenhagen appears to have been the relation of these formal considerations to the more speculative hopes placed in the Feynman quantization approach, especially by Wheeler. Wheeler (1957, p. 607) had speculated that at scales on the order of the Planck length multiply connected spaces would "contribute importantly to the sum over histories". The question was now how this was to be reconciled with his $\mathrm{PhD}$ student's cautious formal attempts to construct a measure, which implied a "natural barrier" to the integration domain. This question was discussed extensively in Copenhagen, but with no definite outcome. Here, the division between DeWitt's and Wheeler's approaches clearly showed (see also Sect. 4.5): While Wheeler wanted to tease out the extreme and conceptually interesting features of a quantum theory of gravity, DeWitt repeatedly insisted that the formal questions needed to be settled first ${ }^{65}$.

Given this formal bent, it is no wonder that DeWitt had found himself in Lille just a week before the Copenhagen workshop ${ }^{66}$. Here, from June 3-8, 1957, a conference on mathematical problems of quantum field theory had been held that is widely regarded as one of the founding events of axiomatic and algebraic field theory, and even of modern mathematical physics as a subdiscipline of physics ${ }^{67}$. Here, it appears, DeWitt had spoken with Julian Schwinger, his former PhD advisor, about another quantization procedure to be applied to general relativity, Schwinger's own action

\footnotetext{
${ }^{62}$ The power of $5 / 2$ essentially arises as number of independent components of the metric (10) times rank of the metric tensor (2) over (dimension of spacetime (4) times weight of the metric determinant (2)).

${ }^{63}$ Misner, in a letter to Wheeler of 10 August 1957 (JAWP, Box 18, Misner correspondence), reporting on the workshop, stated that he "had some difficulty understanding" Laurent's arguments.

${ }^{64}$ This dismissal was accepted by Misner, as he outlines in his interview with Rickles and Salisbury, see footnote 49.

${ }^{65}$ DeWitt was far more explicit on the divide between his views and Wheeler's in the first version of the report, where he wrote: "[I]n contrast to Professor Wheeler's present views $[\ldots]$ we should prefer to work with a simple topology and a simple integration domain". (DF, Box "Institute of Field Physics", unnamed folder 7)

${ }^{66}$ DeWitt is not listed in the official list of participants of the conference reprinted in (Fredenhagen 2010), but the full report contains one comment by DeWitt in discussions (NN 1959). Schwinger is listed as a participant (though with the wrong initial), but his talk appears not to have gone well (see Mehra and Milton 2000, p. 381, and letter from Källén to Pauli of 10 July 1957, printed in von Meyenn 2005).

${ }^{67}$ See (Fredenhagen 2010), Wightman's remarks in (von Meyenn 2005, p. 1059, fn 4), and (Haag 2010, p. 274).
} 
principle. As opposed to the covariant and Feynman quantization, Schwinger's method had not arisen from an attempt to solve the difficulties of quantum field theory. Rather, it had been developed by Schwinger in the early 1950s in response to the great success of Feynman's methods in quantum electrodynamics, as an attempt to put Feynman's heuristic quantization procedure on surer mathematical footing ${ }^{68}$. As Misner had pointed out in his article on Feynman quantization, it had not yet been applied to gravity.

It is hardly surprising that DeWitt, champion of a pluralist approach to quantum gravity, took the next step and quizzed the master himself on the potential of the Schwinger method in quantum gravity. But DeWitt appears not to have been particularly impressed with this potential: Schwinger's method just did not seem practical enough and indeed had not yet shown its merits by leading to new physical results (as opposed to the other three quantization methods discussed above and below). The main point discussed in Copenhagen regarding the Schwinger method was that it required ${ }^{69}$ one to cast the field theory to be quantized into a form where the Lagrangian was only a first-order polynomial in the derivatives of the field variables. Such a reformulation existed for general relativity, using the connections as independent dynamical variables in addition to the metric, and goes by the name of Palatini formalism ${ }^{70}$.

But this was not much discussed in Copenhagen, making the question of Schwinger quantization a mere appendix to the discussion of Feynman quantization. The only issue discussed in any detail was a "curious ambiguity", which probably refers to a difficulty first discussed by Weyl (1950): In the regular Palatini formalism, the usual relations between connection and metric appear as the Euler-Lagrange equations obtained from varying the connection variables. This is spoiled, however, by the inclusion of Dirac spinor fields, minimally coupled to the gravitational field using tetrads and infinitesimal rotations of the local tetrad frames in place of metric and connection. As Weyl had pointed out equivalence between the Palatini and the regular metric formulation could be re-established by adding to the Dirac-Einstein-Hilbert Lagrangian in the regular formulation an additional term quartic in the spinor field ${ }^{71}$. But even this discussion of an interesting aspect of Schwinger quantization and the use of the Palatini formalism it implied does not seem to have led to any novel conclusions in Copenhagen.

${ }^{68}$ See a letter from Freeman Dyson to Rudolf Peierls of 23 September 1950: "He [Schwinger] translates the Feynman Lagrangian formalism with "integration over histories" into a rigorous and conventional language. [...] You have to search carefully how [this] is hidden in the notes. Of course, the name of Feynman is never mentioned. Only I happen to know from other sources (as is also obvious when you see what Schwinger's method actually is) that Schwinger started the whole thing from making Feynman's method intelligible to himself". The letter is reproduced in (Lee 2009).

69 This is not quite how DeWitt expresses it in his report, and indeed formally the Schwinger method could be applied to Lagrangians involving higher powers of the field derivatives. Indeed, several years before, the Syracuse group had attempted to apply Schwinger's method to general relativity in the usual formulation, only to run into the factor ordering complications discussed by DeWitt (Bergmann and Schiller 1953, section 5). Schwinger himself by the late 1950s regarded the construction of a first-order formulation as an essential prerequisite for his method. This is witnessed by references to the Schwinger method in papers by former Schwinger students, e.g., (Arnowitt and Deser 1959) or, in a non-quantum-gravity context, (Glashow 1959).

70 Despite having been developed by Einstein himself, see (Ferraris et al. 1982).

71 The highly plausible identification of the "curious ambiguity" with the difficulty discovered by Weyl was made by Stanley Deser in an email to one of the authors (AB) of 22 March 2016. 
In the years immediately following the Copenhagen workshop, however, the Schwinger approach would deliver much more fruitful results than the Feynman one: Just one year later, Deser and another Schwinger student, Richard Arnowitt, began to tackle the quantization of gravity using the Palatini formalism and Schwinger quantization, soon joining forces with Misner (who had, after finishing his thesis, abandoned the idea of Feynman quantization) to construct, not a quantum theory of gravity, after all, but the ADM formalism ${ }^{72}$.

\subsection{Canonical quantization}

As DeWitt writes in the report, the canonical quantization of the gravitational field was at the time a business primarily pursued by the group of Peter Bergmann in Syracuse. Bergmann's research program had been initiated in the late 1940s as a conservative alternative to Einstein's unified field theory program (on which Bergmann had worked in the 1930s and 1940s), a research program based on the established structures of both general relativity and quantum theory, in particular on the "tried and (so far) true method" of canonical quantization applied to the classical field theory of general relativity. The first order of business had been a reformulation of that classical theory in canonical (Hamiltonian) form, which had been achieved around 1950 in a two-step process: First, the Syracuse group had constructed a Hamiltonian formulation of general relativity with full four-dimensional symmetry (Bergmann et al. 1950), using the so-called parameter formalism. In a second step, they had then turned to (and successfully constructed) a 3+1-decomposed formulation, due to the difficulties of the four-dimensional formulation, both calculational and conceptual (Penfield $1951)^{73}$.

Several years later, in 1957, Syracuse was still the hub of research in canonical quantum gravity, even though several of Bergmann's graduate students had turned away from the problem of quantum gravity and immersed themselves in problems within general relativity proper, such as gravitational waves or the problem of motion - a characteristic shift in focus of the renaissance period, which we have already observed in the case of Misner and the Wheeler school. Bergmann on several occasions had formulated what he took to be the central outstanding issue in the canonical quantization program, namely the identification of the "true observables". This question arose because of the general covariance of the Einstein field equations, which Bergmann had identified early on as the essential feature of general relativity that he believed would carry over into a quantum theory of gravity. General covariance implied that in the canonical formulation of the theory not all of the phase-space variables could be taken as independent dynamical quantities. Rather, there existed in the classical theory non-dynamical identities involving the phase-space variables, the so-called constraints, which had to be fulfilled in addition to the equations of motion. There were eight such constraints in total, four "primary" (arising directly from the defining relations for the canonical momenta), and four "secondary" (arising from the demand that the primary constraints be conserved in time). These constraints had been identified by the Syracuse group in 1950/51, along with the Hamiltonian, thereby establishing a full canonical formulation of general relativity.

The general setup had been quite familiar already in 1950 from quantum electrodynamics, where a similar, though less involved, situation occurred due to gauge

\footnotetext{
72 See (Deser 2015).

${ }^{73}$ On this early history of the canonical approach to quantum gravity, see (Blum and Rickles 2017, section 5).
} 
invariance. In QED, the next step had now traditionally ${ }^{74}$ been to solve the constraints, leading to a modified Hamiltonian that now only contained independent physical degrees of freedom; in the case of QED, these were the transverse modes of the electromagnetic field (photons). This elimination of redundant degrees of freedom had, in QED, originally been performed only after quantization (Fermi 1932; Oppenheimer 1930), but it had later been established that this could also been done pre-quantization, identifying the elimination of the constraints as the choice of a certain gauge, the Coulomb or (in the absence of charged matter) radiation gauge (Heitler 1944).

After the establishment of the Hamiltonian formulation of general relativity in $1950 / 51$, it was clear to Bergmann that the next step should be an elimination of the constraints, in analogy to (quantum) electrodynamics.

[B] uild up the Hilbert space from only those states that satisfy all constraints and $[\ldots]$ make it, thus, deliberately, a small subspace of the functional space of all conceivable wave functionals without regard to constraints, [...] severely restricting the number of linear operations that may properly be called Hilbert operators. [...]

Will the elimination of a large number of operations from consideration not embarass us as physicists, by eliminating the mathematical description of physically meaningful quantities?

In answer, we find that the observables not ruled out are invariants, quantities that remain unchanged under all infinitesimal transformations with respect to which the theory is assumed to be invariant. For electrodynamic quantities, for instance, commutability with the subsidiary conditions of quantum electrodynamics implies gauge invariance. And truly, any quantity that can be given a well-defined numerical value must be an invariant.

We have gone through a number of examples to convince ourselves that any physically observable quantity is an invariant, but this point is so obviously of major importance that it should be more fully investigated. Suffice it here to say that the point of view we have adopted is a generalization of and consistent with accepted practices in quantum electrodynamics and elsewhere. (Bergmann and Schiller 1953, pp. 12-13)

At the 1955 Pisa conference on elementary particle physics, Bergmann had introduced the name "true observables" for these invariant quantities, the operators corresponding to which should be the only ones operating in the reduced Hilbert space of constraint-obeying wave functionals. He further asserted that:

It would appear that particularly in theories possessing general covariance the determination of the true observables is a necessary preliminary to their quantization. Unfortunately this determination remains so far an unsolved problem.

(Bergmann 1956, p. 1178)

Bergmann thus believed that in quantum gravity one did not have the liberty of eliminating the constraints before or after quantization, as in quantum electrodynamics. Rather, one was forced to perform the reduction of the degrees of freedom already pre-quantization. In order to understand how Bergmann came to this conclusion, it is important to realize that "general covariance" for him was a somewhat wider notion that encompassed all those properties of general relativity which he considered essential and worthy of conserving in the transition to the quantum theory. And besides the invariance with respect to general coordinate transformations, this also meant

74 This procedure had been replaced in the 1950s by more sophisticated, covariant techniques, such as the Gupta-Bleuler method. 
the non-linearity of the theory. Such a theory would in its Hamiltonian and its constraints include terms non-linear in both the canonical coordinates and the canonical momenta and there was no unambiguous symmetrization procedure for transferring such an expression into the quantum theory. Indeed, for any given quantization rule for such expressions, it was to be expected that the commutator algebra of the constraints and the Hamiltonian would not close, destroying the self-consistency of the theory. This was the so-called factor ordering problem, and Bergmann hoped to avoid it by finding the true observables before quantizing:

Once the observables have been determined, then presumably any Hermitian combination, which in the limit $\hbar \rightarrow 0$ goes over into the classical Hamiltonian represents a formally possible quantum theory. Then it will be necessary to develop new physical (rather than formal) criteria for the appropriate factor sequence. (Bergmann and Goldberg 1955, p. 538)

DeWitt had been interested in Bergmann's program early on. Initially, as a graduate student, he had approached the problem of quantum gravity from the perspective of the new covariant methods in quantum field theory, developed in the late 1940s by, among others, DeWitt's advisor Julian Schwinger. This approach - as discussed in Sections 2.1 and 2.2 - fundamentally relied both on perturbation theory and on Lorentz covariance, implying as a starting point not the full Einstein equations, but rather the linear approximation, supplemented as necessary with higher-order non-linear correction terms. As his thesis work was contemporaneous with the successful establishment of the Hamiltonian formulation of full general relativity by the Bergmann group (and the Toronto/Pittsburgh group consisting of Alfred Schild and his graduate students Felix Pirani and - later - Ray Skinner), the paper based on his thesis which he submitted to the Physical Review in 1950, had been effectively rejected as too long and detailed given its purely approximative approach. DeWitt had wholeheartedly accepted this criticism and delved into the canonical approach to quantum gravity, writing two papers on canonical quantum gravity, dealing, respectively, with the factor ordering problem (DeWitt 1952) and the inclusion of spinorial quantum fields (DeWitt and DeWitt 1952) ${ }^{75}$.

DeWitt's work was based on the early Hamiltonian formulations, which displayed full four-dimensional symmetry, using the parameter formalism first developed by Paul Weiss in the $1930 \mathrm{~s}^{76}$. As already mentioned, this formalism was soon abandoned by both the Syracuse and the Pittsburgh groups: Not only were the calculations difficult, it was also unclear physically how to interpret the fact that the space-time coordinates (as functions of the auxiliary parameters) had to be taken as non-commuting qnumbers, in addition to the metric tensor field. This step of abandoning the parameter formalism was, however, not followed by DeWitt, who briefly left the field in 1952, at about the time that the Pittsburgh group turned to the non-parameterized $3+1$ formulation (see section 2.2). Returning to his work on quantum gravity in 1956, the interconnections between different approaches to the problem became a leitmotif for DeWitt. Initially focusing on the Feynman sum-over-histories (path integral) approach (see the preceding section), he returned to working on the canonical quantum gravity program after the 1957 Chapel Hill conference he had organized ${ }^{77}$. Here, DeWitt

\footnotetext{
$\overline{75}$ See (Blum and Rickles 2017).

76 On the history and details of this formalism, see (Rickles and Blum 2015).

77 The strong interconnection that DeWitt observed in his work on different quantum gravitational formalisms is also witnessed by his brief remark that his work on the canonical approach would shed light on the problem of the "true measure" arising in the path integral approach, by, it is assumed, identifying the true dynamical degrees of freedom.
} 
picked up right where he had left things in 1952, using the parameterized formalism that had been abandoned elsewhere ${ }^{78}$.

This anachronism was no problem in Copenhagen, where no representatives of the cutting edge of canonical quantization were present. Bergmann's (and Wheeler's) presence had originally been anticipated ${ }^{79}$, but the final roster saw not a single (even former) member of the Syracuse group. This absence of experts in the field of constrained Hamiltonian dynamics, however, also implied that the details of DeWitt's work did not make much of a splash. In an informal report which Misner wrote for DeWitt, but which he only quoted in the first version of the report, not the final one, Misner stated that

I learned to understand the basic ideas of canonical quantization of general relativity, which had escaped me on several previous attempts at reading the published research in this problem ${ }^{80} \ldots$

But, as we will see later, it would take a second exposure for the relevance of these ideas to really sink in. Misner would later recall:

I don't think I absorbed then the Dirac style primary/secondary constraint ideas Bryce gives in the report ${ }^{81}$.

What then was this work of DeWitt's? There had already for some time been the hope that one might find a canonical transformation in the Hamiltonian formulation of general relativity that would turn (some of) the constraint equations into "pure momenta" that is into the form "one canonical momentum variable equal to zero". One would then quite easily be able to eliminate the constraint and the canonical momentum variable in question (along with its conjugate canonical coordinate) from the theory. Jim Anderson (who had been a PhD student of Bergmann's) recalls having thought along these lines already in the summer of $1954^{82}$ and the Copenhagen report supports this view, naming Anderson as the one to have "conjectured the possibility of such a transformation". In Copenhagen, DeWitt now presented his derivation of just such a transformation which turned the primary constraints of the theory into pure momenta, thereby making a big step towards solving the central difficulties of the canonical quantization program outlined above.

The open question was whether such a transformation into pure momenta also existed for the secondary constraints, thereby completing the elimination of constraints in the theory. DeWitt could present no final results concerning this question, only a general theorem which stated that such a transformation would exist only if the Lie

\footnotetext{
${ }^{78}$ It should be noted that DeWitt was well aware of the later developments in the canonical theory. Indeed, he often uses terminology that really only makes sense in the unparameterized formalism. In particular, when he speaks of primary constraints, he means only those primary constraints that also show up in the unparameterized formalism. The additional primary constraints appearing in the parameterized formalism DeWitt does not attempt to eliminate. After all, this would simply be deparameterizing. We will adopt his terminology - hence, in the following, primary constraints refers only to the four primary constraints associated with canonical momenta for the metric variables.

${ }^{79}$ As witnessed by a letter from Klein to Wheeler of 20 April 1957, Relativity Notebook $\mathrm{V}$, JAWP.

${ }^{80}$ First version of the 1957 Copenhagen report. DF, Box "Institute of Field Physics", unnamed folder 7 .

81 Email to one of the authors (AB) of 1 April 2016.

${ }^{82}$ Interview with Jim Anderson by Dean Rickles and Donald Salisbury, 19 March 2011.
} 
group generated by the subset of constraints being eliminated ${ }^{83}$ was Abelian $^{84}$. But he was unable at the time to determine whether, in fact, the Lie group generated by the secondary constraints was Abelian, due to the underdeveloped state of the mathematical theory of "infinite Lie groups" 85 .

But, as already mentioned, these exciting developments within the canonical quantization program hardly made an impression on DeWitt's audience. Rather, their main focus appears to have been on DeWitt's outdated mathematical tools, namely the parameter formalism. Whence this interest in DeWitt's parameters? On the one hand, the emphasis on the parameter formalism in the report appears to be due to DeWitt's own interests. He had apparently convinced himself that the abandonment of the parameter formalism by the other groups working on canonical quantization had been misguided. In particular, he believed that the use of the parameter formalism would help in finding the canonical transformation that turned the secondary constraints into pure momenta.

This claim of DeWitt's is somewhat hard to assess. He appears to indeed have eliminated the primary constraints in the parameterized formalism and to have found the Hamiltonian and the secondary constraints in the now reduced phase space ${ }^{86}$. DeWitt's "Hamiltonian" (Equation 3) looks somewhat curious, with its free Lorentz index - for any specific choice of parameterization, a specific linear combination of DeWitt's $\varphi_{\mu}$, which are just are the constraints derived from the defining relations for the momenta canonically conjugate to the space-time coordinates, would be singled out as the actual Hamiltonian. This splitting up of the Hamiltonian makes the calculation of the secondary constraints somewhat cumbersome (16 Poisson brackets need to be calculated, for only four secondary constraints), but it does the job. The resultant secondary constraints of the parameterized formalism have some unusual features; in particular they are, as opposed to the unparameterized case, linear in the momenta, which gave DeWitt some hope, that they, too, could be transformed into pure momenta. And in a grant proposal to the Research corporation one year later ${ }^{87}$ DeWitt would in fact claim that he was able to show quite generally that in the parameterized theory the Lie group generated by both the primary and the secondary

\footnotetext{
83 Since the commutator/Poisson bracket of two constraints is a linear combination of constraints, the constraints can be viewed as elements of a Lie algebra.

${ }^{84}$ Apparently Anderson had conjectured that such a transformation would always exist for the primary constraints of the theory and that the only problem was to find it. DeWitt now stated that this only happened to be the case for general relativity, where the algebra generated by the primary constraints was indeed Abelian.

${ }^{85}$ That is, in particular, gauge symmetries. As DeWitt points out, the importance of such groups had also been stressed by Wolfgang Pauli in a series of lectures on group theory that he had given for the theory division of CERN in Copenhagen in September 1955 (Pauli 1965, p. 86). The lecture notes were first published as a CERN report in 1956, so they were certainly available in Copenhagen in Summer 1957. Pauli's emphasis was likely influenced both by Pauli's engagement with non-abelian gauge theories in 1953/54 (Straumann 2000) and with his work on updating his book on general relativity in 1955/56 (von Meyenn 2001, pp. $498 \mathrm{ff})$.

${ }^{86}$ Both Bergmann (with Johanna Brunings) and Pirani and Schild had originally falsely believed that there were only primary constraints in the parameterized theory (Blum and Rickles 2017).

${ }^{87}$ Since Wheeler was one of DeWitt's references, this proposal can be found in the John Archibald Wheeler papers, Box 7, DeWitt correspondence. DeWitt's signature is dated 5 June 1958.
} 
constraints was Abelian and that he had thereby managed to turn the secondary constraints into pure momenta as well ${ }^{88}$. But he went on:

Having completed this stage of the work, however, DeWitt is now running into new difficulties. Because of the complexity of the equations, he is finding it very difficult to remove all of the non-observable quantities from the Hamiltonian of the system. Part of the trouble is that he now, at this point, has a lack of general principles which would guide him and enable him to see the forest instead of the trees. Stimulated by his modest success with the aid of group theoretical principles, DeWitt is now undertaking an investigation of the theory of infinite dimensional Lie groups.

DeWitt had thus finally, despite all his computational prowess, run into the same difficulties of complexity that had led others to abandon the parameter approach almost a decade earlier. A first publication on infinite dimensional Lie groups followed three years later (DeWitt 1961) and made no more reference to the parameterized formalism, leaving a test of the validity of DeWitt's claims as a challenge to contemporary physicists. Similarly, it is an open question how DeWitt's remarks on the nonlocality of the true observables (and especially the speculative footnote on the possibility of obtaining fermionic spinors) are to be interpreted in the usual, non-parameterized form of the theory ${ }^{89}$.

In any case, as already mentioned several times, there was no-one at the Copenhagen workshop who could or wanted to delve into these details of constrained Hamiltonian dynamics. The main interest of the other participants appears to be summarized in the last paragraph of this section, the only place where a name other than DeWitt's is mentioned. The question being discussed was what was later coined the "problem of time", that is the vanishing of the Hamiltonian in General Relativity. In the parameterized formalism, the Hamiltonian always vanished, for the reasons that DeWitt discussed: It generates the dynamics based on the parameter $T$ rather than on the physical time. This was well-known and was initially believed to be an artefact of the parameterized formalism which would vanish in the non-parameterized $3+1$ formulation, as stated, e.g., by Bergmann and his PhD student Ralph Schiller:

$[\mathrm{T}]$ he introduction of parameters [...] does not lead to serious modifications. Most important, the Hamiltonian becomes itself a constraint. There is, therefore, no "motion". Any state that obeys all constraints is a solution of the Schrödinger equation, provided we do not permit it to change in the course

88 The authors have not been able to determine whether the proposal was accepted. Certainly Wheeler's lukewarm recommendation did not help. On 9 December 1958, he wrote to the Research Corporation (JAWP, Box 7, DeWitt correspondence): "I would not be honest if I tried to indicate that DeWitt is one of the top three investigators in the field of general relativity in the world; he would be more like number six in this country". This was not, it should be added, Wheeler's last word on the matter. Another two years later, on 29 November 1960, he would write to the Chairman of the Department of Physics at the University of North Carolina (ibid.): "I would say that no one in the world has at the same time a wider command of the mathematical techniques of relativity theory plus quantum theory - together with the drive to carry through more complex calculations to a definitive end than does Bryce DeWitt".

89 Though DeWitt was certainly not alone at the time with his conjecture concerning the non-locality, see, e.g., a letter from Bergmann to Pauli of 17 November 1957 (Bergmann Papers, Syracuse), where he states that he had also believed that the true observables in general relativity should be non-local. Note that this letter is not reprinted in the Pauli Correspondence volumes, despite the fact that Bergmann appears to have sent it to the editors. 
of "time". This apparent freezing is, however, a purely formal result of the introduction of parameters. (Bergmann and Schiller 1953, p. 13)

In the following years, however, the suspicion began to emerge that also in the nonparameterized Hamiltonian formulation of general relativity, where the Hamiltonian density gives the dynamics based on the coordinate time $t$, the Hamiltonian would vanish, as remarked again by Bergmann in the closing sentence of (Bergmann and Goldberg 1955) and discussed at the 1957 Chapel Hill conference (DeWitt and Rickles 2011, p. 191 and p. 231). The question also arose in Misner's PhD thesis on the path integral quantization of general relativity, where he had derived the Hamiltonian density operator $\mathcal{H}_{\mathrm{op}}$ appearing in the Schwinger-Tomonaga (relativistic Schrödinger) equation and found it to be zero. Misner went on to write that:

Because of different methods of definition, our $\mathcal{H}_{\text {op }}$ Hamiltonian is not necessarily the operator corresponding to the Hamiltonian defined in classical theory. However Professor J.L. Anderson at the Chapel Hill conference voiced suspicions that the classical Hamiltonian in general relativity would be zero. (Misner 1957, p. 508)

It appears now that at the Copenhagen workshop a consensus was reached that indeed Misner's Hamiltonian operator did not correspond to the classical Hamiltonian, but was in effect the Hamiltonian of the parameterized formalism, which vanished trivially. Misner's work thus had no bearing on the actual "problem of time", which then was not discussed any further in Copenhagen. This view clearly was adopted by Misner himself after Copenhagen: When Wolfgang Pauli wrote to him later in 1957, inquiring as to what the physical implications of the vanishing of Misner's Hamiltonian was, he replied that it was a mere triviality:

It comes down to saying that $[. .$.$] the Schwinger-Tomonaga equation considers$ variations that are as meaningless physically as coordinate transformations, and therefore produce no change in the state-vector. I think you, Bergmann and I all agree that a Hamiltonian density, if defined in this way, must vanish ${ }^{90}$.

While this was the main upshot of DeWitt's presentations on canonical quantization in Copenhagen, his work would make a bigger splash several months later, when he presented it to a more receptive audience at the Stevens Institute of Technology in Hoboken, NJ. His host, Jim Anderson (1958), immediately picked up on DeWitt's work and presented the canonical transformation that allowed for the elimination of the primary constraints in the non-parameterized formalism just two months later. Anderson's work was, however, soon marginalized, as Paul Dirac (1958) had obtained the same results at exactly the same time (though, it appears, independently of both DeWitt and Anderson) and taken them quite a bit further. But another member of the audience at the Stevens Institute was now a lot more receptive to DeWitt's presentation than he had been in Copenhagen: Charles Misner.

According to Misner's recollections (which are based on the detailed notebooks he kept in the months after the Copenhagen workshop), he had turned in the fall of 1957 to the wormhole problem (see sect. 3.2):

After the 57 summer I started a research notebook (Wheeler style) on 9 November 1957 when an ONR (Office of Naval Research) grant through the Math Department in Princeton [...] was supporting my research. There [...] I turned to trying to make Wheeler's "wormhole" sketch rigorous. One step was to find the initial conditions, done by early January 1958. The next step would

\footnotetext{
${ }^{90}$ Letter from Misner to Pauli, 19 November 1957, Bergmann Papers, Syracuse. Note that this letter is not reprinted in the Pauli Correspondence volumes.
} 
be to numerically evolve it in time. A page long note from an IBM session outlines on 31 December 1957 what this involves, including the question of how to tell the computer to lay out coordinates in the future as time progresses beyond the initial conditions. Both the coordinate choice problem, and how to take a step forward in time were studied ${ }^{91}$.

Hearing DeWitt's presentation at the Stevens meeting, Misner now realized that this problem was closely related to DeWitt's work on eliminating constraints ${ }^{92}$. And several months later, Misner was really bringing his expertise on the matter to bear on what would become known as the ADM collaboration, culminating in the 1962 review article that is now primarily cited (Arnowitt et al. 1962).

\subsection{Approximation methods}

This section (we will return to the question of the idiosyncratic title in due time) deals mainly with the work of Deser (and Laurent) on rendering the quantum field theories of the non-gravitational (electromagnetic and nuclear) interactions finite by including the effects of the quantized gravitational field. While attributed to Klein by DeWitt, the origin of the idea is rather to be found in the work of Lev Landau and Wolfgang Pauli.

Landau's involvement in the measurability debate with Bohr and Rosenfeld in the early 1930s has already been discussed in Section 4.1. After this defeat, Landau essentially abandoned quantum electrodynamics for two decades, returning to it only in 1953, instigated by his young collaborators Alexei Abrikosov and Isaac Khalatnikov (Abrikosov 1973). Landau originally believed that they could, by using an approximation technique that went beyond the usual perturbation theory, prove that QED was in fact a perfectly finite theory, even as one let the momentum cutoff $\Lambda$ go to infinity. But soon he realized a sign mistake and had to draw an entirely different conclusion: His approximation was in fact inconsistent for large cutoffs (and a fortiori for infinite cutoffs, i.e., for a fully Lorentz-invariant theory) (Ioffe 2012). But rather than viewing this as a mere defect of his approach, Landau revived the attack against relativistic quantum field theory that he had dropped twenty years before, and in a follow-up paper written together with Isaac Pomeranchuk - head of the theory division at the Moscow Institute for Theoretical and Experimental Physics and the leader of Soviet QFT research up until Landau's re-entering the field - drew much more radical conclusions from the anomaly (nowadays known as "Landau Pole") that he had identified, namely that the physical (renormalized) charge in QED would always be zero, independent of the value of the bare (unrenormalized) charge, that, in other words, QED was a non-interacting quantum field theory (Landau and Pomeranchuk $1965)$.

These views reached Wolfgang Pauli in Switzerland through several channels indeed Landau had worked hard to communicate his ideas to Western physicists, as this slowly became possible in the years after Stalin's death. Landau had invited Pauli's Swedish collaborator Gunnar Källén to a Moscow conference on QED in the Spring of 1955 (Jarlskog 2014) and also wrote a contribution to the Festschrift celebrating Bohr's 70th birthday in 1955 (Pauli 1955), a contribution that the editors (which included Pauli) had solicited without really expecting it to materialize ${ }^{93}$. Pauli was quite happy to hear of Landau's views, which coincided with the conclusions he

\footnotetext{
91 Email to one of the authors (AB) of 1 April 2016.

${ }^{92}$ Interview with Misner and Dieter Brill by Dean Rickles and Don Salisbury, 16 March 2011.

${ }^{93}$ Letter from Léon Rosenfeld to Pauli of 24 May 1954, published in (von Meyenn 1999).
} 
himself had drawn from his recent investigations into the structure of renormalized quantum field theory with Källén:

I am of course satisfied to see that he [Landau] has the same suspicions concerning quantum electrodynamics that I do. [...] From what I have read of the paper, it seems probable to me, however, that he has as little proof for this conjecture as I do. [...] So it appears to me: Landau's "nose" is still good, but mathematical proofs were never his strong suit ${ }^{94}$.

But what intrigued Pauli most, was Landau's attempt to connect the failure of QED with gravitation. As Landau had written in paper for the 1955 Bohr Festschrift:

Of course, no unambiguous physical conclusions can be drawn from the result obtained, that the point [i.e., infinite cutoff] interaction is zero in the case of electrodynamics. The energies $\Lambda[\ldots]$ are in every case very large. At these energies, the effects of gravitational interaction may exceed the electromagnetic effects, so that a discussion of electrodynamics as a closed system becomes physically incorrect. The idea is very attractive that this "crisis" in electrodynamics occurs for just those energies where the gravitational interaction is comparable with the electromagnetic. (Landau 1955, p. 60)

Landau gave no indication as to how gravitation might solve the zero charge crisis. Neither did Pauli. But he did have some ideas on how the inclusion of gravitation might affect quantum field theory in general. And just a few months after hearing of Landau's arguments, Pauli had the opportunity to present his ideas on the relevance of general relativity to field theory to the most interested audience imaginable, at the 1955 Bern relativity conference. This underlines one more time the catalytic role of the 1955 conference, both in bringing older physicists back to general relativity Pauli of course having made his fame as a young man with a review article on general relativity - and introducing younger physicists to the theory. For in the audience was a young postdoc with a training in Schwingerian quantum field theory, but hardly any prior exposure to general relativity: Stanley Deser.

Pauli presented his ideas in the discussion following the talk by Oskar Klein. Klein was another older physicists, who had been prompted to return to research on relativity by the upcoming Bern meeting ${ }^{95}$. He had been working on the five-dimensional theory (that now of course bears his name, along with that of Theodor Kaluza) on and off, ever since his pioneering work in the mid-1920s. In his Bern lecture (Klein 1956), Klein, too, now spoke of the connection between quantum electrodynamics and general relativity. His early work on the five-dimensional theory had been concerned with establishing the connections between general relativity and the newly emerging wave mechanics, and he was now attempting to do a similar thing with the new renormalized quantum electrodynamics, a theory he was only now really starting to learn ${ }^{96}$. Klein's main argument for such a connection was that the discretization of the electric charge, obtained by making the wave functions of charged matter waves periodic in the fifth coordinate, should be regarded as "a quantum condition in classical disguise" (p. 59). He emphasized that the ratio of the Planck length (which appears in the "ordinary quantisation of gravitation theory", p. 61) and the periodicity length (which gave the "quantization" of the electric charge) was just the fine structure constant of QED and concluded that "[t]o have these two processes of quantization connected is the same as to determine the value of" the fine structure constant (Pauli's holy grail,

94 Letter from Pauli to Källén, 24 April 1955, printed in (von Meyenn 2001).

${ }^{95}$ Pauli, as president of the conference, appears to actually have played a role in this, see letter from Klein to Pauli of 6 August 1954, published in (von Meyenn 1999).

96 See letter from Klein to Pauli of 6 August 1954, (von Meyenn 1999). 
incidentally). Klein went on to speculate that

A near lying possibility of such a connection is that the relation between the periodicity length] and [the Planck length] is determined by the renormalisation of the electric charge through vacuum polarisation, which in an adequate theory ought to be finite.

Klein's speculations prompted Pauli to the following remarks in discussion:

$[\mathrm{T}]$ he connection [...] of the mathematical limitation of quantum electrodynamics with gravitation pointed out by LANDAU and KLEIN, seems to me to hint at the indeterminacy in space-time of the theory, invariant with respect to the wider group of general relativity. It is possible that this new situation so different from quantized theories, invariant with respect to the Lorentz group only, may help to overcome the divergence difficulties which are so intimately connected with a c-number equation for the light-cone in the latter theories (p. 69).

Pauli's vague remarks were discussed during the course of 1956 by Deser and Klein ${ }^{97}$, now separated from their original Landau pole context and viewed as a general recipe for eliminating divergences (acting as a regulator) in quantum field theory. These discussions resulted in a paper by Deser (1957), first presented at the Chapel Hill Conference and then published in the Chapel Hill special issue of the Reviews of Modern Physics. In this paper, Deser (acknowledging his discussions with Klein) had managed to flesh out Pauli's ideas. Deser's idea was to use a path-integral approach, which in the case of a quantized gravitational field would also involve an integration over configurations of the metric field. This was supposed to ameliorate the divergences in the following manner: In the usual theory, the propagators (in position space) would exhibit their characteristic divergent behavior on the light cone by involving expressions of the form $1 /\left(x_{\mu}-x_{\mu}^{\prime}\right)^{2}$, a divergent behavior that lay at the heart of the divergences of quantum field theory in general. In a theory with a quantized gravitational field, Deser argued, such a singular expression should be replaced by an expression of the form

$$
\int \mathcal{D} g e^{\frac{i}{\hbar} A_{g}} \frac{1}{\left(\int_{x}^{x} \sqrt{g_{\mu \nu} d x^{\mu} d x^{\nu}}\right)^{2}}
$$

where $\mathcal{D} g$ is the functional integration over the gravitational field variables. The singularity of the usual propagator would thus be removed, since in integrating over the metric configurations there would only be a set of measure zero in which $x$ and $x^{\prime}$ actually lie on each other's light cone.

Deser's ideas may have been a fleshing out of Pauli's brief remarks, but they, too, were still rather vague. At Chapel Hill, Deser consequently received a fair amount of criticism. He recalls that it was particularly the comments by Richard Feynman that convinced him of the "lack of control over the whole regularization scheme" 98 still present in his sketch. The conference proceedings also mention comments by Lichnerowicz and Fourès-Bruhat, remarking on the lacking definition of the measure (see Sect. 4.3), and an unattributed criticism regarding the order of integration: If one integrates over the spacetime coordinates first, before integrating over the gravitational ones, one encounters the usual divergences before gravity can step in to save the day. Deser had answered that this was "partly due to an unallowed interchange of limits", but the sketch he had provided was hardly sufficient to substantiate such an assertion.

97 Email from Stanley Deser to one of the authors (AB), 12 May 2016.

98 Email from Stanley Deser to one of the authors (AB), 24 March 2016. 
The Copenhagen workshop then sees Deser and Laurent, several months after Chapel Hill, trying to gain control of the scheme through approximation methods. The approximations employed were rather radical, as they simply replaced the action $A_{g}$ for the gravitational field, appearing in the phase of the path integrand, which includes both the usual Hilbert action and radiative corrections arising when integrating out the matter fields, by the most simple field action possible, namely the Klein-Gordon action, normally used for a scalar field (multiplied by the inverse of Newton's constant, to make up for the fact that the metric does not have the correct units to be a scalar field $)^{99}$. But even with this radical approximation, they were unable to make progress on the idea, as the path integral was still not of Gaussian (the only calculable) form, due to the metric showing up in the denominator, which was of course central to the proposed elimination of the divergences. The work at Copenhagen can thus be considered the death throes of the gravity as regulator idea; Deser would not work on it again afterwards.

In DeWitt's report, Deser's work was packaged together with Misner and Wheeler's ideas on quantum foam, which they had developed during their time in Leiden one year earlier (see Wheeler and Ford 2000, p. 246-263). Not much work appears to have been done on quantum foam in Copenhagen, and DeWitt's packaging Deser's regulator work and quantum foam together seems somewhat curious at first glance. For sure, both of them relied on path integral heuristics. But, half a year earlier, after Deser's talk at Chapel Hill, Wheeler had rather pointed out the disanalogies, remarking that the effects of functional integration over metric configurations would be far more radical than Deser envisioned, since one would also have to integrate over configurations with different topologies (Wheeler 1957). DeWitt argued for lumping the two together because both of them assumed significant effects of high energy quantum gravity effects in low-energy theories, despite the extreme feebleness of the actual gravitational interaction. In particular, the inclusion of quantum gravitational effects precluded the use of a flat space Minkowski metric as a zeroth order approximation to the structure of space-time (hence, ultimately, the name of the section). For the case of quantum foam this was a vague, physically motivated conjecture, in Deser's case it clearly showed in his approximate expression for the matter propagator: If one here expands the denominator around the Minkowski metric, the leading term is again the usual propagator, light-cone singularities and all.

But concerning the expected effects of high-energy quantum gravity contributions on the low-energy theory, Deser and Wheeler were miles apart. Deser was merely arguing that the elimination of divergences he envisioned would not occur at any finite order in perturbations around a flat metric. The actual contributions to the low-energy propagator were expected to be minute. On the other hand, Wheeler was expecting to explain the actual existence of particles and charge in the low-energy theory as a result of high-energy quantum-gravitational fluctuations. At Chapel Hill, Deser indeed appears to have quite explicitly disagreed with Wheeler on this point. In a note that Wheeler took after Deser's presentation of the regulator work at Chapel Hill, he wrote:

How important is high freq[uency] stuff? JAW [John Archibald Wheeler] says very; Deser says little ${ }^{100}$.

\footnotetext{
99 The fact that the metric is now effectively a scalar field in Deser and Laurent's approximation, is underscored by the fact that it always has lower indices, as a distinction between co- and contravariant is no longer possible.

100 Relativity Notebook V, p. 99, JAWP.
} 
So, it would appear that in this section, DeWitt is really just arguing against the view of the Wheeler school ${ }^{101}$, especially where he states that the non-locality, which Wheeler expected to arise from topological fluctuations at the quantum level, should arise already at the classical level, and would thus directly appear in the quantum theory after the usual quantization and approximation procedures were applied to the canonical formulation of general relativity he was currently constructing (see the previous section). One can only speculate as to why DeWitt would dampen his criticism of Wheeler by formulating it primarily as a criticism of Deser. But certainly DeWitt was professionally very much dependent on Wheeler, who had been the "godfather" of DeWitt's Institute of Field Physics and even at this point had great influence on the funding DeWitt received there (See Footnote 88).

In any case, the question at stake here was really a methodological, rather than a physical one: Should one try to tease out the implications of quantum gravity without actually having such a theory at hand, or should one just press on and try to construct a full quantum gravitational formalism? DeWitt was here arguing for the latter. A very concise formulation of his view can be found in the above-mentioned grant application:

[E]ither one must quantize the gravitational field or one must formulate a new and very basic principle in physics. As there is no indication at present of what this basic principle should be - or as one is not clever enough to bring it to light - it is worth attempting to quantize the gravitational field. Either one will succeed and thus understand the connection between two major theories of physics - the gravitational theory and the quantum theory - or one will fail and learn something from the failure.

\subsection{Some special problems}

The investigation of gravitational radiation had been from the beginning strongly influenced by a close analogy with electromagnetic theory. Einstein's original derivation of gravitational waves had relied on the weak field approximation, and thus involved none of the nonlinear aspects of the gravitational field, where most of its distinctive features were expected to appear. Moreover, gravitational waves - if they existed were expected to be transverse waves traveling at the speed of light, just like their electromagnetic counterparts. Felix Pirani made, in 1962, a remark that summarizes well the resilience of analogies in the research on gravitational radiation:

$[\mathrm{O}]$ ne cannot $[\ldots]$ expect all the familiar attributes of electromagnetic radiation to have analogues in the case of gravitational radiation. [Nevertheless, some] analogy has to be sought, because the concept of radiation is until now largely familiar through electromagnetic theory, and one cannot define gravitational radiation sensibly without some appeal to electromagnetic theory for guidance (Pirani 1962, p. 91, see also Rickles 2010).

But, bit by bit - as mentioned in section 2.1 - the particularities of general relativity began to move to the focus of attention and the analogy began to erode. DeWitt began section 6 of the report with a clear statement in that direction.

\footnotetext{
101 There really does not appear to have been much of a difference of opinion on these matters between Deser and DeWitt. Deser, upon reading DeWitt's report for the first time in 2016, indeed remarked that the discussion hinted at here had made no impression at all and actually voiced his support for DeWitt's stance that "[w]e always have to expand" (Email to one of the authors (AB) of 22 March 2016). Conversely, only seven years after the Copenhagen meeting, DeWitt (1964) would actually bring forth an argument that Deser's speculations about gravity as a regulator were in fact correct.
} 
The Copenhagen meeting was not the first time that DeWitt reflected on the differences between electromagnetic radiation and gravitational radiation. He first thought systematically about these matters when he was preparing a talk for the theoretical physics panel of the Third Annual Meeting of the American Astronautical Society, which took place in New York on 7 December 1956. The panel apparently gathered DeWitt, Peter Bergmann, Freeman Dyson, and Louis Witten, and was chaired by Agnew Bahnson $^{102}$. That talk was DeWitt's first foray into gravitational radiation ${ }^{103}$. There, DeWitt stated:

I would like to give you a glimpse into the subtlety of the necessary analysis [in general relativity] by considering how one would arrive at a unique definition of gravitational radiation. [...] Let's ask how we would, if we could, in principle, measure gravitational radiation. We have to work by analogy. How do we measure electromagnetic radiation? We measure electromagnetic radiation essentially by the jolt it gives us when it passes by us (DeWitt 1957a, p. 24).

The informal style was adapted to the purpose of the talk: it was aimed at publicizing the activities of the new Institute of Field Physics to a broad audience (DeWitt and Rickles 2011, p. 16). DeWitt explained the fundamental difference between electromagnetic and gravitational radiation in the following lines. In order to measure electromagnetic radiation, one must observe a varying force on a charged particle i.e., a third time derivative of position. Since the force is the gradient of the potential, and supposing that one can transform time derivatives into space derivatives using the chain rule, the existence of an electromagnetic radiation is related to the second spatial derivative of the potential.

The measurement of a gravitational radiation is more subtle: "In the gravitational case, you don't feel any jolt because everything gets jolted by the same amount. [...] There is no net jolt. How are you going to measure it? Well, here is the conceptional solution" (DeWitt, 1957b, p. 25). DeWitt imagined the following thought experiment. Two objects are falling in free fall towards a planet. One only knows that there is a gravitational field because these objects approach each other, due to the convergence of the lines of force. Therefore, the existence of a gravitational field can be observed by a measurement of their mutual acceleration. Such an acceleration is the gradient of the gravitational force and, thus, the second spatial derivative of the potential. If one wants to observe a gravitational wave, one must have a variation in the mutual acceleration of those two objects. DeWitt concluded that the measurement of a gravitational wave depends on measuring the third derivative of the potential.

This gives you an idea of how you have to go to a more subtle mathematical stage to reach an useful and invariant definition of gravitational radiation. This shows you an important difference between gravity and electromagnetism, and one which forces many analogies to break down (DeWitt 1957a, p. 25).

Probably DeWitt got the idea for the thought experiment from conversations with John Wheeler. The main experimental efforts in general relativity at that time were led, independently, by Joseph Weber and by Robert Dicke, both working at Princeton. Weber was particularly devoted to measuring gravitational waves, with no success. His research was in all respects guided by analogies with the electromagnetic radiation. At the 1961 Varenna summer school on experimental tests of general relativity, organized

\footnotetext{
$\overline{102}$ Bahnson Memoranda \#5 (16 October 1956). DF, box Institute of Field Physics.

103 In fact, he had discussed gravitational radiation in his $\mathrm{PhD}$ thesis, but in a quite naive way, without paying attention to the differences between the electromagnetic and the gravitational case (DeWitt 1949). Only in the 1956 talk did he reflect on the matter systematically.
} 
by Christian Møller, Weber explained:

Let us for a moment consider the [gravitational wave] problem from the standpoint of an experimentalist. He would really like to do laboratory experiments similar to those which Hertz did on electromagnetic waves almost a century ago, that is, he would like to generate and detect such waves in his laboratory (Weber 1962, p. 116).

The experiment carried out by Weber (1960) used a gravitational antenna (called "mass quadrupole detector") designed in close analogy to the electromagnetic case. While Weber's work initialized the entire field of gravitational wave detection, the analogy with the electromagnetic case was ultimately abandoned in favor of a detection method specific to gravitational waves (based on laser interferometry), culminating in the first detection of gravitational waves in 2015.

The relation between electromagnetic and gravitational radiation was mentioned several times at the Chapel Hill conference. Hermann Bondi expressed a critical perspective: "The analogy between electromagnetic and gravitational waves has often been made, but doesn't go very far, holding only to the very questionable extent to which the equations are similar" (DeWitt and Rickles 2011, p. 95). John Wheeler expressed a more optimistic view. According to him, the richness of Maxwell's theory should act as a source of enthusiasm for the relativists, providing questions and allowing them "to draw new richness out of the [general relativity] theory on the classical level" (DeWitt and Rickles 2011, p. 44). As an example, he mentioned: "[A] task ahead of us is the construction of the curve giving the spectrum of gravitational radiation incident on the earth, analogous to the known curve of the electromagnetic radiation spectrum; or at least the determination of upper limits on it" (DeWitt and Rickles 2011, p. 45). Therefore, Wheeler is not seeking formal analogies between both theories, but rather using electromagnetism merely a general source of inspiration to general relativity.

In section 6 of the Copenhagen report, DeWitt aimed at providing further illustration of the subtle differences between electromagnetic and gravitational radiation. Those examples show, in DeWitt's own words, "in a simple way how radically different the gravitational field is from other fields". Therefore, analogical methods should be used with due care, for gravitation was not just another field theory. This was one of the main conclusions of the Copenhagen meeting.

At the end of section 6, DeWitt refers to Dirac's 1938 article. It may be interesting to say here a little on DeWitt's work immediately after the Copenhagen meeting, since it was done under the influence of that classical article and was related to the radiation reaction issues. One of the questions raised at the Chapel Hill conference as we mentioned in section 4.1 - was: "[C]an the principle of equivalence be extended to elementary particles?" (DeWitt and Rickles 2011, p. 167). DeWitt's second PhD student, Robert Brehme, asked him - probably in late 1957 - a similar question: Does a falling electric charge radiate? As DeWitt recalled:

I was at that time [late 1957] trying to develop a canonical formalism for the gravitational field with the aim of creating a quantum theory of gravity, and I hoped that Brehme would assist me in this work. In fact, the work bogged down in the usual difficulties familiar to anyone who has tried to construct, and make sense of, a canonical quantum theory of gravity. So, in desperation, I agreed to let Brehme investigate the falling-charge problem; but I insisted that he do it properly. He was to begin by studying Dirac's famous 1938 paper on the classical radiation electron, in which all calculations are performed in a manifestly Lorentz invariant manner. He was then to translate this paper into the language of curved spacetime, keeping all the derivations manifestly 
generally covariant. He was not to introduce a special coordinate system at any stage (DeWitt 1996b, p. 34).

DeWitt and Brehme's research exposed for the first time several interesting aspects of radiation reaction in curved spacetimes, including a nice application of Synge's world function (DeWitt and Brehme 1960, see also Poisson et al. 2011, p. 19). In the course of their work, they studied Jacques Hadamard's book in great detail - which allowed DeWitt to master Green functions in curved spacetimes, a technique that turned out to be a fundamental ingredient in the covariant approach to quantum gravity that he would develop during the 1960s (DeWitt 1962a, 1965, 1967; Hadamard 1923).

\section{Conclusions}

As we hope to have shown through our contextualization and analysis, Bryce DeWitt's Copenhagen report not only provides a fascinating and invaluable overview of the state of quantum gravity in 1957, it also presents DeWitt's vision of establishing quantum gravity as a field of research within the emerging general relativity community of the renaissance. DeWitt's vision was an inclusive one: He had worked on perturbative approaches as a Ph.D. student, and in Copenhagen he sought to bring together canonical and Feynman quantization approaches, to open up a dialogue and to establish connections. He also sought to bring the older generation on board, which was more interested in epistemological questions of measurement and uncertainty relations. The question we might now ask is, given our observation in the introduction that to this date there is no unified research field of quantum gravity, whether the Copenhagen meeting was a success.

At first glance one may well answer in the affirmative: DeWitt made substantial progress on his parameterized, canonical approach; Møller, DeWitt, and Misner explored the role of clocks in establishing the consistency of a quantum theory of the gravitational field; Misner's and Laurent's different approaches to the measure in Feynman quantization began to converge; and Deser and Misner would soon begin to collaborate (together with Richard Arnowitt) on the Schwinger quantization of gravitation. But if we trace these developments only a little bit further, we realize that all of these hopeful beginnings soon turned into dead ends: DeWitt abandoned the canonical approach soon after, just as Misner abandoned his work on Feynman quantization. Laurent soldiered on, but without making much progress. DeWitt had promised, in the first version of the report, two papers by himself as an outcome of the Copenhagen workshop (one of them together with Laurent); they were never published. And while the ADM work provided important breakthroughs concerning the concept of energy in general relativity, or the most efficient formulation of the initial value problem (especially for numerical calculations), it was clear to the authors already in the early 1960s that it would not deliver the sought-after quantum theory of gravity. In the first version of the report, DeWitt had written:

A formalism, which is something we do not even yet have in the case of quantum gravidynamics, must come first. Fortunately, it now seems as if we may have one in the not too distant future ${ }^{104}$.

DeWitt had meant that the formalism "must come first" before the questions of measurability could be pursued in earnest. But his statement equally well applies to the question of community-building. The renaissance of general relativity had only been possible because there was a solid theoretical and formal basis on which the entire

104 First version of the 1957 Copenhagen report. DF, Box "Institute of Field Physics", unnamed folder 7 . 
community could agree, the Einstein equations at the very least. There appeared to be in Copenhagen a convergence and an emerging understanding between the various approaches, but as each of them ran into their own individual difficulties, this budding consensus soon faded. DeWitt had envisaged a future in which a core formalism of quantum gravity would soon emerge (most probably in the best-defined approach, the canonical one). The different approaches would then be viewed as different perspectives on the same theory - as had happened in the history of quantum electrodynamics in the late 1940s. The various research agendas would then merge into a common field of quantum gravity research. So, e.g., even though he had dismissed Schwinger's action principle as a way of constructing the core formalism, he welcomed it as a future way of analyzing that formalism. As he wrote in the report:

It seemed highly unlikely to the participants at Copenhagen that Schwinger's methods would really prove useful in the search for the hidden path to a quantum theory of gravitation. On the other hand, there is no doubt that when a quantum gravitational formalism is finally found Schwinger will be on hand to reformulate it.

But DeWitt's plans were frustrated. The uncontroversial core formalism (to be found at the end of the "hidden path") that had been DeWitt's objective in 1957 soon receded beyond the horizon and with it the memory of the Copenhagen workshop. Despite the fact that the origins of ADM can in many ways be traced to the Copenhagen meeting, Deser would later state that the Copenhagen meeting (as opposed to the conferences in Bern and Chapel Hill) had left "no traces" in his memory ${ }^{105}$ and Misner also stated that he had "almost no physics recollections of the 1957 workshop"106. Rather than becoming a joint research field, quantum gravity gradually turned into a race, if it can be called that, with the various competitors running off in different directions, apparently indifferent to one another. As the different approaches moved apart, it became increasingly difficult to translate from one to the other.

DeWitt would make substantial progress in the decade after Copenhagen, culminating in his famous 1967 trilogy. He continued to contribute to the canonical approach (the Wheeler-DeWitt equation), but in the years after 1957 mainly focused on a new approach that essentially reversed the view he had held in Copenhagen. While he had then defended the position that discussions of measurement would come after the formalism, he now pursued the idea that the consistent measurability demands could actually define the formalism. But he pursued this path alone and without achieving ultimate success.

Essentially to this day, quantum gravity remains what DeWitt had fought so hard for it not to be: A high-energy phantom theory that could liberally be employed for heuristic investigations of measurability questions, the ultimate constitution of matter (Wheeler), or the consistency of low-energy quantum field theories (as in Deser's regulator attempts). We hope that the reader may draw some inspiration from the glimpse the Copenhagen report provides of the brief moment in time when there was a unified quantum gravity community.

Acknowledgements. We would like to thank Wolf Beiglböck, Stanley Deser, Domenico Giulini, Roberto Lalli, and Dean Rickles for valuable comments on the manuscript. We also thank Cécile DeWitt-Morette, the Niels Bohr Archive (Copenhagen), the Niels Bohr Library and Archives (College Park), and the American Philosophical Society (Philadelphia) for their authorization to consult their collections. T.H. thanks Olival Freire Jr., Reinaldo de

\footnotetext{
105 Email to one of the authors (AB) of 22 March 2016.

106 He did remember meeting his future wife in Copenhagen in the summer of 1957. Email to one of the authors (AB) of 23 March 2016.
} 
Melo e Souza, and Finn Aaserud for conversations and advice; the funding agencies CNPq (Brazil) and Novo Nordisk Foundation (Denmark); and the Friends of the Center for History of Physics (American Institute of Physics) for a grant-in-aid which funded his research at the Niels Bohr Library and Archives. Open access funding provided by Max Planck Society.

\section{References}

Abrikosov, A.A. (1973). My years with Landau. Physics Today 26: 56-60.

Anderson, J.L. (1958). Reduction of primary constraints in generally covariant field theories. Physical Review 111: 965-966.

Arnowitt, R. and S. Deser. (1955). Renormalization of derivative coupling theories. Phys. Rev. 100(1):349-361.

Arnowitt, R. and S. Deser. (1959). Quantum theory of gravitation: General formulation and linearized theory. Phys. Rev. 113: 745-750.

Arnowitt, R., S. Deser and C.W. Misner. (1962). The dynamics of general relativity. In Witten, L., editor, Gravitation: An Introduction to Current Research. Wiley, New York, Chap. 7, pp. 227-265.

Ashtekar, A. (2014). The last 50 years of general relativity and gravitation: from GR3 to GR20 Warsaw conferences. General Relativity and Gravitation 46: 1706 (1-17).

Bergmann, P.G. (1942). Introduction to the Theory of Relativity. Prentice-Hall, New York.

Bergmann, P.G. (1956). Introduction of "true observables" into the quantum field equations. Il Nuovo Cimento III: 1177-1185.

Bergmann, P.G. and I. Goldberg. (1955). Dirac bracket transformations in phase space. Phys. Rev. 98: 531-538.

Bergmann, P.G., R. Penfield, R. Schiller and H. Zatzkis. (1950). The Hamiltonian of the general theory of relativity with electromagnetic field. Phys. Rev. 80: 81-88.

Bergmann, P.G. and R. Schiller. (1953). Classical and quantum field theories in the lagrangian formalism. Phys. Rev. 89: 4-16.

Bhabha, H.J., editor (1951). Report of an international conference on elementary particles, Bombai. Tata Institute/UNESCO.

Blum, A., R. Lalli and J. Renn. (2015). The reinvention of general relativity: A historiographical framework for assessing one hundred years of curved space-time. Isis 106: 598-620.

Blum, A.S. and D. Rickles, editors (2017). Quantum Gravity in the First Half of the XXth Century: A Sourcebook. Edition Open Access, Berlin.

Bohr, N. (1928). The Quantum Postulate and the Recent Development of Atomic Theory. Nature 121: $580-590$.

Bohr, N. and L. Rosenfeld. (1933). Zur Frage der Messbarkeit der elektromagnetischen Feldgrößen. Det Kgl. Danske Videnskabernes Selskab Mathematisk-fysiske Meddelelser 12 (8): 1-65.

Brevik, I.H. (2011). Christian Møller: The concepts of mass and energy in the general theory of relativity I-II (DKNVS Forhandlinger 1958). Det Kongelige Norske Videnskabers Selskabs Skrifter, pp. 93-102.

Bronstein, M. (1936). Quantentheorie schwacher Gravitationsfelder. Physikalische Zeitschrift der Sowjetunion 9: 140-157.

Darrigol, O. (1991). Cohérence et complétude de la mécanique quantique: l'exemple de "Bohr-Rosenfeld". Revue d'histoire des sciences 44: 137-179.

Deser, S. (1957). General relativity and the divergence problem in quantum field theory. Rev. Mod. Phys. 29: 417-423.

Deser, S. (1977). Oskar Klein. Phys. Today 30: 67-68.

Deser, S. (1987). Gravity from self-interaction in a curved background. Classical and Quantum Gravity 4: L99-L105.

Deser, S. (1995). Oskar Klein: from his life and physics. Technical Report TH-95-09, CERN.

Deser, S. (2015). The legacy of ADM. Physica Scripta 90: 068006 (4pp). 
Deser, S. and A. Starobinsky. (2012). Editorial note to: Matvei P. Bronstein, Quantum theory of weak gravitational fields. General Relativity and Gravitation 44: 263-265.

DeWitt, B. (1949). I: The Theory of Gravitational Interactions. II: The Interaction of Gravitation with Light. Ph.D. thesis, Harvard.

DeWitt, B. (1952). Point transformations in quantum mechanics. Phys. Rev. 85: 653-661.

DeWitt, B. (1957a). Principal directions of current research activity in the theory of gravitation. Journal of Astronautics 4: 23-28.

DeWitt, B. (1957b). Dynamical theory in curved spaces. I. A review of the classical and quantum action principles. Rev. Mod. Phys. 29: 377-397.

DeWitt, B. (1961). Quantization of fields with infinite-dimensional invariance groups. $J$. Math. Phys. 2: 151-162.

DeWitt, B. (1962a). The quantization of geometry. In Witten, L., editor, Gravitation: An Introduction to Current Research. Wiley, New York, Chap. 8, pp. 266-381.

DeWitt, B. (1962b). Quantization of fields with infinite-dimensional invariance groups. III. Generalized Schwinger-Feynman theory. J. Math. Phys. 3: 1073-1093.

DeWitt, B. (1964). Gravity: A universal regulator? Phys. Rev. Lett. 13: 114-118.

DeWitt, B. (1965). Dynamical Theory of Groups and Fields. Gordon and Breach, New York.

DeWitt, B. (1967). Quantum theory of gravity. II. The manifestly covariant theory. Phys. Rev. 162: 1195-1239.

DeWitt, B. (1971). The Many-Universes Interpretation of Quantum Mechanics. In d'Espagnat, B., editor, Fondamenti di meccanica quantistica: Rendiconti della scuola internazionale di fisica Enrico Fermi. Academic Press, New York, pp. 211-262.

DeWitt, B. (1996a). Preliminary remarks before beginning his technical talk. In Ng, Y.J., editor, Julian Schwinger: The Physicist, the Teacher, and the Man. World Scientific, Singapore, pp. 29-31.

DeWitt, B. (1996b). The uses and implications of curved-spacetime propagators: A personal view. In Ng, Y.J., editor, Julian Schwinger: The Physicist, the Teacher, and the Man, pages 33-59. World Scientific, Singapore.

DeWitt, B. (2003). The Global Approach to Quantum Field Theory. Oxford University Press, New York.

DeWitt, B. and R.W. Brehme. (1960). Radiation damping in a gravitational field. Ann. Phys. 9: 220-259.

DeWitt, B. and C.M. DeWitt. (1952). The quantum theory of interacting gravitational and spinor fields. Phys. Rev. 87: 116-122.

DeWitt, C.M. (2011). The Pursuit of Quantum Gravity: Memoirs of Bryce DeWitt from 1946 to 2004. Springer, Berlin.

DeWitt, C.M. and Rickles, D., editors (2011). The Role of Gravitation in Physics: Report from the 1957 Chapel Hill Conference. Edition Open Access, Berlin.

Dirac, P.A.M. (1932). Relativistic quantum mechanics. Proc. Roy. Soc. Lond. A136: 453464.

Dirac, P.A.M. (1950). Generalized Hamiltonian dynamics. Canadian J. Math. 2: 129-148.

Dirac, P.A.M. (1958). The theory of gravitation in Hamiltonian form. Proc. Roy. Soc. Lond. A246: 333-343.

Einstein, A. (1953). The Meaning of Relativity (Fourth Edition, including the Generalization of Gravitation Theory). Princeton University Press, Princeton.

Einstein, A., L. Infeld and B. Hoffmann. (1938). The gravitational equations and the problem of motion. Ann. Math. 39: 65-100.

Einstein, A. and N. Rosen. (1935). The particle problem in the general theory of relativity. Phys. Rev. 48: 73-77.

Eisenstaedt, J. (1986). La relativité générale à l'étiage: 1925-1955. Archive for History of Exact Sciences 35: 115-185.

Fermi, E. (1932). Quantum theory of radiation. Rev. Mod. Phys. 4: 87-132.

Ferraris, M., M. Francaviglia and C. Reina. (1982). Variational formulation of general relativity from 1915 to 1925 "Palatini's Method" discovered by Einstein in 1925. General Relativity and Gravitation 14: 243-254.

Feyerabend, P. (1995). Killing Time. University of Chicago Press, Chicago. 
Feynman, R.P. (1950). Mathematical formulation of the quantum theory of electromagnetic interaction. Phys. Rev. 80: 440-457.

Feynman, R.P. (1963). Quantum theory of gravitation. Acta Physica Polonica 24: 697-722.

Feynman, R.P., F.B. Moringo and W.G. Wagner. (1995). Feynman Lectures on Gravitation. Addison-Wesley, Reading, MA.

Fierz, M. and W. Pauli. (1939). On relativistic wave equations for particles of arbitrary spin in an electromagnetic field. Proc. Roy. Soc. Lond. A173: 211-232.

Fisher-Hjalmars, I. and B. Laurent. (1991). Oskar Klein. In Ekspong, G., ed. The Oskar Klein Memorial Lectures. World Scientific, Singapore, Vol. 1, pp. 1-9.

Fourès-Bruhat, Y. (1952). Théorème d'existence pour certains systèmes d'équations aux dérivées partielles non-linéaires. Acta Mathematica 88: 141-225.

Fredenhagen, K. (2010). Lille 1957: The birth of the concept of local algebras of observables. Eur. Phys. J. H 35: 239-241.

Glashow, S.L. (1959). The renormalizability of vector meson interactions. Nucl. Phys. 10: $107-117$.

Goldberg, J.N. (1992). US Air Force support of general relativity: 1956-1972. In Eisenstaedt, J. and Kox, A., editors, Studies in the History of General Relativity, volume 3 of Einstein Studies. Birkhäuser, Boston, pp. 89-102.

Goldberg, J.N. and E.L. Schucking. (2003). Peter Gabriel Bergmann. Phys. Today 56: 64-66.

Gupta, S.N. (1952a). Quantization of Einstein's gravitational field: general treatment. Proc. Phys. Soc. 65: 608-619.

Gupta, S.N. (1952b). Quantization of Einstein's gravitational field: linear approximation. Proc. Phys. Soc. A 65: 161-169.

Gupta, S.N. (1954). Gravitation and electromagnetism. Phys. Rev. 96: 1683-1685.

Haag, R. (2010). Some people and some problems met in half a century of commitment to mathematical physics. Eur. Phys. J. H 35: 263-307.

Hadamard, J. (1923). Lectures on Cauchy's problem in linear partial differential equations. Yale University Press, New Haven.

Halpern, P. (2004). The Great Beyond: Higher Dimensions, Parallel Universes, and the Extraordinary Search for a Theory of Everything. Wiley.

Hartz, T. and O. Freire. (2015). Uses and appropriations of Niels Bohr's ideas about quantum field measurement, 1930-1965. In Aaserud, F. and Kragh, H., editors, One hundred years of the Bohr atom: Proceedings from a conference, volume 1 of Scientia Danica. Series M, Mathematica et physica, pages 397-418, Copenhagen. Det Kongelige Danske Videnskabernes Selskab.

Heisenberg, W. (1938). Die Grenzen der Anwendbarkeit der bisherigen Quantentheorie. Zeitschrift für Physik 110: 251-266.

Heisenberg, W. and W. Pauli. (1929). Zur Quantendynamik der Wellenfelder. Zeitschrift für Physik 56: 1-61.

Heitler, W. (1944). The Quantum Theory of Radiation, 2rd edn. Oxford University Press, Oxford.

Iliopoulos, J. (1996). Physics in the CERN theory division. In Krige, J., editor, History of Cern - Volume 3. North Holland, Amsterdam, Chap. 8, pp. 277-326.

Infeld, L., editor (1964). Relativistic Theories of Gravitation: Proceedings of a conference held in Warsaw and Jablonna, July 1962, London. Pergamon Press.

Infeld, L. and A. Schild. (1949). On the motion of test particles in general realtivity. Rev. Mod. Phys. 21: 408-413.

Ioffe, B.L. (2012). The first dozen years of the history of ITEP theoretical physics laboratory. arXiv: $1208.1386 \mathrm{v} 1$.

Jacobsen, A.S. (2011). Crisis, measurement problems, and controversy in early quantum electrodynamics: The failed appropriation of epistemology in the second quantum generation. In Kojevnikov, A., Carson, C., and Trischler, H., editors, Quantum Mechanics and Weimar Culture: Revisiting the Forman Thesis. Imperial College Press, London, pp. 375-396.

Jarlskog, C. (2014). Portrait of Gunnar Källén, chapter 52: At 1955 Moscow Meeting. Springer, Switzerland, pp. 223-225. 
Kalckar, J. (1971). Measurability problems in the quantum theory of fields. In d'Espagnat, B., editor, Proceedings of the International School of Physics "Enrico Fermi". Academic Press, New York, pp. 127-168.

Kalckar, J., editor (1996). Niels Bohr Collected Works, Volume \%. North-Holland, Amsterdam.

Klauder, J. and J.A. Wheeler. (1957). On the question of a neutrino analog to electric charge. Rev. Mod. Phys. 29: 516-517.

Klein, O. (1955). Quantum theory and relativity. In Pauli, W., editor, Niels Bohr and the Development of Physics. Pergamon Press, London, pp. 96-117.

Klein, O. (1956). Generalisations of Einstein's theory of gravitation considered from the point of view of quantum field theory. In Mercier, A. and Kervaire, M., editors, Jubilee of Relativity Theory - Proceedings, pages 58-71, Basel. Birkhäuser.

Kosmann-Schwarzbach, Y. (2009). Tribute to André Lichnerowicz (1915-1998). Notices of the AMS 56: 244-246.

Kraichnan, R.H. (1955). Special-relativistic derivation of generally covariant gravitation theory. Phys. Rev. 98: 1118-1122.

Krige, J. (1987a). Case studies of some important decisions. In Hermann, A., Krige, J., Mersits, U., and Pestre, D., editors, History of Cern - Volume 1. North Holland, Amsterdam, Chap. 8, pp. 237-292.

Krige, J. (1987b). Survey of development. In Hermann, A., Krige, J., Mersits, U., and Pestre, D., editors, History of Cern - Volume 1. North Holland, Amsterdam, Chap. 7, pp. 209236.

Krige, J. (2006). American Hegemony and the Postwar Reconstruction of Science in Europe. MIT Press, Cambridge, MA.

Landau, L. (1955). On the quantum theory of fields. In Pauli, W., editor, Niels Bohr and the Development of Physics. Pergamon Press, London.

Landau, L. and R. Peierls, (1931). Erweiterung des Unbestimmtheitsprinzips für die relativistische Quantentheorie. Zeitschrift für Physik 69: 56-69.

Landau, L. and I. Pomeranchuk. (1965). On point interactions in quantum electrodynamics. In ter Haar, D., editor, Collected Papers of L. D. Landau. Clarendon Press, Oxford, pp. 654-658.

Lee, S., editor (2009). Sir Rudolf Peierls: selected private and scientific correspondence. World Scientific, New Jersey, Vol. 2.

Lichnerowicz, A. (1944). L'intégration des équations de la gravitations relativiste et le problème des n corps. J. Math. Pures Appl. 23: 37-63.

Lichnerowicz, A. (1955). Théories Relativistes de la Gravitation et de l'Électromagnétisme. Masson, Paris.

Lichnerowicz, A. (1964). Propagateurs et quantification en relativité générale. In Infeld, L., editor, Relativistic Theories of Gravitation: Proceedings of a conference held in Warsaw and Jablonna, July 1962, pp. 177-188.

Lichnerowicz, A. (1992). Mathematics and general relativity: A recollection. In Eisenstaedt, J. and Kox, A., editors, Studies in the History of General Relativity, volume 3 of Einstein Studies. Birkhäuser, Boston, pp. 103-108.

Lichnerowicz, A. and Tonnelat, M., editors (1962). Les Théories Relativistes de la Gravitation, Paris. CNRS.

Mehra, J. and Milton, K.A. (2000). Climbing the Mountain: The Scientific Biography of Julian Schwinger. Oxford University Press, Oxford.

Mercier, A. (1992). General relativity at the turning point of its renewal. In Eisenstaedt, J. and Kox, A., editors, Studies in the History of General Relativity, volume 3 of Einstein Studies. Birkhäuser, Boston.

Mercier, A. and Kervaire, M., editors (1956). Fünfzig Jahre Relativitätstheorie. Helvetica Physica Acta, Supplementum IV.

Misner, C.W. (1955). Applications of topology to general relativity. Phys. Rev. 99: 662.

Misner, C.W. (1957). Feynman quantization of general relativity. Rev. Mod. Phys. 29: 497509. 
Misner, C.W. and Wheeler, J.A. (1957). Classical physics as geometry. Ann. Phys. 2: 525603.

Møller, C. (1952). The Theory of Relativity. Clarendon Press, Oxford.

Nambu, Y. (1950). The use of the proper time in quantum electrodynamics. I. Progress of Theoretical Physics 5: 82-94.

NN, editor (1959). Colloque International du Centre National de la Recherche Scientifique. Les problèmes mathématiques de la théorie quantique des champs., Paris.

Oppenheimer, J.R. (1930). Note on the theory of the interaction of field and matter. Phys. Rev. 35: 461-477.

Pauli, W., editor (1955). Niels Bohr and the Development of Physics. Pergamon Press, London.

Pauli, W. (1965). Continuous groups in quantum mechanics. Ergebnisse der exakten Naturwissenschaften 37: 85-104.

Penfield, R.H. (1951). Hamiltonians without parameterization. Phys. Rev. 84: 737-743.

Pestre, D. (1987). The period of conflict, August-December 1951. In Hermann, A., Krige, J., Mersits, U., and Pestre, D., editors, History of Cern - Volume 1. North Holland, Amsterdam, Chap. 5, pp. 147-177.

Pirani, F. (1962). Survey of gravitational radiation theory. In $\mathrm{n} / \mathrm{a}$, editor, Recent Developments in General Relativity. Pergamon Press, New York, Chap. 6, pp. 89-105.

Pirani, F.A.E. and A. Schild. (1950). On the quantization of Einstein's gravitational field equations. Phys. Rev. 79: 986-991.

Poisson, E., A. Pound and I. Vega. (2011). The motion of point particles in curved spacetime. Living Reviews in Relativity, 7.

Rickles, D. (2010). Quantum gravity meets \&HPS. In Mauskopf, S. and Schmaltz, T., editors, Integrating History and Philosophy of Science: Problems and Prospects. Springer, Dordrecht, pp. 163-199

Rickles, D. and A.S. Blum. (2015). Paul Weiss and the genesis of canonical quantization. Eur. Phys. J. H 40: 469-487.

Rosenfeld, L. (1930a). Zur Quantelung der Wellenfelder. Ann. Phys. 397: 113-152.

Rosenfeld, L. (1930b). Über die Gravitationswirkungen des Lichtes. Zeitschrift für Physik 65: $589-599$.

Rovelli, C. (2004). Quantum Gravity. Cambridge University Press, Cambridge.

Rozental, S. (1985). NB: Erindringer om Niels Bohr. Gyldendal, Copenhagen.

Salecker, H. and Wigner, E. (1958). Quantum limitations of the measurement of space-time distances. Phys. Rev. 109: 571-577.

Salisbury, D. and Sundermeyer, K. (2016). Léon Rosenfeld's invention of constrained Hamiltonian dynamics. arXiv:1606.06076.

Schucking, E.L. (1989). The first Texas symposium on relativistic astrophysics. Phys. Today 42: $46-52$.

Schweber, S.S. (1994). QED and the Men Who Made It: Dyson, Feynman, Schwinger, and Tomonaga. Princeton Univ Press, Princeton.

Schwinger, J. (1948). Quantum electrodynamics. I. A covariant formulation. Phys. Rev. 74: $1439-1461$.

Solomon, J. (1938). Gravitation et quanta. Journal de Physique et Le Radium 9: 479-485.

Straumann, N. (2000). On Pauli's invention of non-Abelian Kaluza-Klein theory in 1953. arXiv: gr-qc/0012054v1.

ter Haar, D., editor (1965). Collected Papers of L. D. Landau. Pergamon Press, London.

Tomonaga, S. (1946). On a relativistically invariant formulation of the quantum theory of wave fields. Progress of Theoretical Physics 1: 27-42.

von Borzeszkowski, H.-H. and H.-J. Treder. (1988). The Meaning of Quantum Gravity. Springer, Netherlandds.

von Meyenn, K., editor (1993). Wolfgang Pauli: Wissenschaftlicher Briefwechsel mit Bohr, Einstein, Heisenberg u.a., volume III: 1940-1949. Springer, Berlin.

von Meyenn, K., editor (1996). Wolfgang Pauli: Wissenschaftlicher Briefwechsel mit Bohr, Einstein, Heisenberg u.a., volume IV/Part I: 1950-1952. Springer, Berlin. 
von Meyenn, K., editor (1999). Wolfgang Pauli: Wissenschafticher Briefwechsel mit Bohr, Einstein, Heisenberg u.a., volume IV/Part II: 1953-1954. Springer, Berlin.

von Meyenn, K., editor (2001). Wolfgang Pauli: Wissenschaftlicher Briefwechsel mit Bohr, Einstein, Heisenberg u.a., volume IV/Part III: 1955-1956. Springer, Berlin.

von Meyenn, K., editor (2005). Wolfgang Pauli: Wissenschafticher Briefwechsel mit Bohr, Einstein, Heisenberg u.a., volume IV/Part IV: 1957-1958. Springer, Berlin.

Weber, J. (1960). Detection and generation of gravitational waves. Phys. Rev. 117: 306-313.

Weber, J. (1962). Theory of methods for measurement and production of gravitational waves. In Møller, C., editor, Evidence for Gravitational Theories (Proceedings of the International School of Physics "Enrico Fermi"). Academic Press, New York, Chap. 4, pp. 116-140.

Weiss, P. (1938). On the Hamilton-Jacobi theory and quantization of a dynamical continuum. Proc. Roy. Soc. Lond. A169: 102-119.

Weyl, H. (1950). A remark on the coupling of gravitation and electron. Phys. Rev. 77: 699701.

Wheeler, J.A. (1955). Geons. Phys. Rev. 97: 511-536.

Wheeler, J.A. (1957). On the nature of quantum geometrodynamics. Ann. Phys. 2: 604-614.

Wheeler, J.A. and R.P. Feynman. (1945). Interaction with the absorber as the mechanism of radiation. Rev. Mod. Phys. 17: 157-181.

Wheeler, J.A. and K. Ford. (2000). Geons, Black Holes \& Quantum Foam. W. W. Norton, New York.

Wigner, E. (2002). Concept of observation in quantum mechanics. In Podolsky, B., Hart, J.B., and Werner, F.G., editors, Conference Manuscript: Conference on the Foundations of Quantum Mechanics (1962). Book 1, Monday Evening Session. Xavier University Exhibit.

Wüthrich, A. (2010). The Genesis of Feynman Diagrams. Springer, Dordrecht.

Zeh, H. (2011). Feynman's interpretation of quantum theory. Eur. Phys. J. H 36: 63-74.

Open Access This is an open access article distributed under the terms of the Creative Commons Attribution License (http://creativecommons.org/licenses/by/4.0), which permits unrestricted use, distribution, and reproduction in any medium, provided the original work is properly cited. 